PLEASE NOTE: This is the Authors' Accepted Manuscript version. The final publication is available at Springer via http://dx.doi.org/10.1007/s10878-012-9511-x.

Citation: Kellerer, Hans, Soper, Alan J. and Strusevich, Vitaly A. (2013) Preemptive scheduling on two identical parallel machines with a single transporter. Journal of Combinatorial Optimization, 25 (2). pp. 279-307. ISSN 1382-6905 (Print), 1573-2886 (Online) (doi:10.1007/s10878-012-9511-x) 


\title{
Preemptive Scheduling on Two Identical Parallel Machines with a Single Transporter
}

\author{
Hans Kellerer \\ Institut für Statistik und Operations Research, Universität Graz, \\ Universitätstraße 15, A-8010, Graz, Austria \\ e-mail: hans.kellerer@uni-graz.at \\ Alan J. Soper and Vitaly A. Strusevich \\ School of Computing and Mathematical Sciences, University of Greenwich, \\ Old Royal Naval College, Park Row, Greenwich, London SE10 9LS, U.K. \\ e-mail: \{A.J.Soper,V.Strusevich\}@greenwich.ac.uk
}

\begin{abstract}
We consider a scheduling problem on two identical parallel machines, in which the jobs are moved between the machines by an uncapacitated transporter. In the processing preemption is allowed. The objective is to minimize the time by which all completed jobs are collected together on board the transporter. We identify the structural patterns of an optimal schedule and design an algorithm that either solves the problem to optimality or in the worst case behaves as a fully polynomial-time approximation scheme.
\end{abstract}

Keywords: Scheduling with transportation $\cdot$ Parallel machines $\cdot$ Approximation scheme

\section{Introduction}

We consider a processing system that consists of two identical parallel machines. The jobs are delivered to the system by a single transporter, moved between the machines by that transporter, and on their completion are transported away. This paper is a complete version of that by Kellerer et al. (2010), where due to the format of that publication not all proofs were included.

Integrating scheduling and logistics decision-making into a single model can be seen as one of the current trends of scheduling theory. In these enhanced models it is required to combine typical scheduling decisions with various logistics decisions, normally related to inventory control, machine breakdowns, maintenance, and various transportation issues.

In the scheduling literature there are several approaches that address the issue of scheduling with transportation. Normally, transportation occurs between the processing stages, and therefore more often than not the processing system is a multi-stage or shop system, e.g., the flow shop and the open shop. Recall that for two machines, e.g., denoted by $A$ and $B$, in the case of the flow shop each job is first processed on machine $A$ and then on machine $B$, while for the open shop, the processing route of each job is not known in advance. In both shop models, each job is seen as consisting of two operations, and the operations of the same job are not allowed to overlap. As a rule, for the problems considered and reviewed in this paper the objective is to minimize the completion time of all jobs on all machines.

In our study, we focus on approximability issues, which have been a topic of considerable interest in the area. A polynomial-time algorithm that creates a schedule with an objective 
function value that is at most $\rho \geq 1$ times the optimal value is called a $\rho$-approximation algorithm; the value of $\rho$ is called a worst-case ratio bound. A family of $\rho$-approximation algorithms is called a fully polynomial-time approximation scheme (FPTAS) if $\rho=1+\varepsilon$ for any $\varepsilon>0$ and the running time is polynomial with respect to both the length of the problem input and $1 / \varepsilon$.

Reviews of four known types of scheduling models with a transportation component can be found in the papers by Lee and Strusevich (2005) and Lushchakova et al. (2009). Two of these types (the robotic cells and the transportation networks) appear to be less relevant to this study and are not discussed below.

The model with Transportation Lags is the most studied among those that combine scheduling with transportation. Here it is assumed that there is a known time lag between the completion of an operation and the start of the same job on the machine that is next in the processing route. These lags can be interpreted as transportation times needed to move a job between the machines, provided that the transportation device is always available. A detailed review of the complexity results on open shop and flow shop scheduling with transportation lags is given by Brucker et al. (2004). For the general case with job-dependent transportation lags, the two-machine open shop problem is unary $N P$-hard even if for any job the durations of its operations are equal. A $\frac{3}{2}-$ approximation algorithm for this problem is due to Strusevich (1999). The two-machine flow shop problem is unary NP-hard even if all processing times are unit, see Yu et al. (2004); several 2-approximation algorithms are given by Dell'Amico (1996). A polynomial-time approximation scheme for the classical flow shop problem developed by Hall (1998) can be modified to handle the transportation lags.

The model that we study in this paper belongs to the class of Models with Interstage Transporters. For the two-machine flow shop the general model of this type is introduced by Lee and Chen (2001). Assume that there are $v$ transporters each capable of carrying $c$ jobs between the machines. The transportation time from $A$ to $B$ is equal to $\tau$, while the travel time of an empty transporter from $B$ to $A$ is equal to $\sigma$. The problem with $c=1$ and $\sigma=0$ is shown to be unary NP-hard by Hurink and Khust (2001). The problem with $v=1$ and $c \geq 3$ is unary NP-hard, while the case of $c=2$ is open, see Lee and Chen (2001). The open shop version of this problem is addressed by Lee and Strusevich (2005) and Lushchakova et al. (2009).

There are no known approximation results for these models, apart from the two-machine flow shop and open shop with a single uncapacitated transporter, i.e., $v=1$ and $c \geq n$. For both the flow shop and open shop models, it is assumed that the jobs are brought by the transporter to one of the machines, moved between the machines in batches, and when the processing is over, the transporter collects the jobs together and carries them away. For this model, the objective is to minimize the time by which all completed jobs are collected on board the transporter.

The classes of heuristic flow shop schedules in which the jobs are split in at most $b$ batches on each machine are studied by Lee and Strusevich (2005) for $b=2$ and by Soper and Strusevich (2007) for $b=3$, and $\frac{b+1}{b}$-approximation algorithms are designed, these ratios being the best possible as long as a heuristic schedule contains at most $b$ batches. For the open shop counterpart of the above problem with $\tau=\sigma$ a $\frac{7}{5}-$ approximation algorithm is developed by Lushchakova et al. (2009).

The problem that we study in this paper belongs to the same family, and its main features are as follows. There are two identical parallel machines, $M_{1}$ and $M_{2}$. The processing time of a job $j \in N=\{1,2, \ldots, n\}$ is equal to $p_{j}$. At time zero, the jobs are brought to the system by a transporter. Each job is either processed without preemption on one of the machines or its 
processing is split into several portions. In the latter case, the total duration of the portions of a job $j$ is still equal to $p_{j}$. For a job to be (partially) processed on a machine, it must be delivered there by the transporter. A move of the transporter between the machines takes $\tau$ time units, and the number of jobs transferred by a move can be arbitrary. Extending the notation adopted by Lee and Strusevich (2005), we call this problem $T P 2|v=1, c \geq n| K_{\max }$, where $K_{\max }$ is the time by which all completed jobs are collected together on board the transporter.

We are aware of only one other study that combines scheduling on parallel machines with transportation, namely the paper by Qi (2006). As in our case, Qi's model also involves two parallel identical machines. However, there are several points of difference between the two models. First, the type of transportation used by Qi is essentially a transportation lag. Second, there is no preemption allowed in Qi's model. Third, the jobs are known to be assigned to the machines in advance and are moved only to be processed on the other machine and returned to the originally assigned machine.

The remainder of this paper is organized as follows. In Section 2 we establish properties of schedules that are optimal for problem $T P 2|v=1, c \geq n| K_{\max }$, and derive lower bounds on the optimal length. Section 3 describes and analyzes an algorithm that creates a schedule with an even number (either two or four) of moves of the transporter, while the case of the schedules with three moves is considered in Section 4. Section 5 presents an approximation algorithm that outputs a non-preemptive schedule. Some concluding remarks are contained in Section 6.

In all NP-hardness proofs the following decision problem is used.

Partition: Given positive integers $e_{1}, \ldots, e_{r}$ and $E$ such that $\sum_{j=1}^{r} e_{j}=2 E$, does there exist a partition of the index set $R=\{1, \ldots, r\}$ into two subsets $R_{1}$ and $R_{2}$ such that $\sum_{j \in R_{1}} e_{j}=\sum_{j \in R_{2}} e_{j}=E$ ?

It is well known that PARTITION is NP-complete in the ordinary sense.

\section{Feasible Schedules: Properties, Structure and Lower Bounds}

In this section, we describe properties of optimal schedules for problem $T P 2 \mid v=1, c \geq$ $n \mid K_{\max }$, identify possible shapes of their structures and establish lower bounds on the optimal value of the objective function.

In problem $T P 2|v=1, c \geq n| K_{\max }$ the jobs of a set $N=\{1,2, \ldots, n\}$ have to be processed on any of two identical processing machines, $M_{1}$ and $M_{2}$. The processing time of a job $j \in N$ is equal to $p_{j}$. Since the machines are identical, we may assume that all jobs are brought by the transporter to machine $M_{1}$ at time zero, so that the first move of the transporter is made from machine $M_{1}$ to machine $M_{2}$. Sometimes we refer to machine $M_{1}$ as the top machine and to machine $M_{2}$ as bottom machine; also the transporter will be said to move down if it moves from $M_{1}$ to $M_{2}$, and to move up otherwise. On their arrival, some of the jobs will be left at $M_{1}$ to be processed and totally completed on that machine. Some other jobs will be moved to machine $M_{2}$ to be processed and totally completed on that machine. There may be jobs that are partly processed on $M_{1}$ and partly on $M_{2}$; however each such job has to be transported to the corresponding machine for (partial) processing. The transporter can move any number of jobs at a time, and the length of a move in either direction is equal to $\tau$ time units. While 
a job is being transported it cannot be processed on either machine; besides, it is not allowed to process a job on both machines simultaneously. The objective is to minimize the length of a schedule, i.e., the time $K_{\max }$ by which all completed jobs are collected together on board the transporter.

For problem $T P 2|v=1, c \geq n| K_{\max }$, let $S^{*}$ denote an optimal schedule, i.e., $K_{\max }\left(S^{*}\right) \leq$ $K_{\max }(S)$ for all feasible schedules $S$. There are three possible types of a feasible schedule $S$ :

Type 0: all jobs are processed on one machine $M_{1}$;

Type 1: the number of moves of the transporter in $S^{*}$ is odd (upon their completion the jobs are collected at machine $M_{2}$ );

Type 2: the number of moves of the transporter in $S^{*}$ is even (upon their completion the jobs are collected at machine $\left.M_{1}\right)$.

In what follows, we assume that a Type 0 schedule is not optimal; otherwise, the problem is trivial.

Given a schedule $S$, the set of jobs that are processed only on machine $M_{1}$ (or on machine $M_{2}$ only) is denoted by $W_{1}(S)\left(W_{2}(S)\right.$, respectively). Notice that set $W_{i}, i \in\{1,2\}$, includes the jobs that are processed on machine $M_{i}$ without preemption, as well as those jobs that are processed on $M_{i}$ in several portions, but not on the other machine. Let $Q(S)$ denote the set of jobs processed with preemption, partly on machine $M_{1}$ and partly on machine $M_{2}$. If no confusion arises, we may drop the reference to a schedule and write $W_{1}, W_{2}$ and $Q$.

If in a schedule $S$ some job $j$ is fractional, i.e., $j \in Q(S)$, then we denote by $x_{j}$ and $y_{j}$ the total length of the time intervals during which job $j$ is processed on machine $M_{1}$ and machine $M_{2}$, respectively. Obviously, $x_{j}+y_{j}=p_{j}$. For a non-empty set $H \subseteq N$ of jobs, denote $p(H):=\sum_{j \in H} p_{j}$ and for completeness define $p(\varnothing):=0$. In a similar sense, we use the pieces of notation $x(H), y(H)$, etc.

Definition 1 For problem $T P 2|v=1, c \geq n| K_{\max }$, a class of feasible schedules in which (i) the first move of the transporter starts at time zero, and (ii) the last move does not transfer any jobs that need to be completed is called Class $\mathcal{S}$.

The following two lemmas show that the search for an optimal schedule can be reduced to schedules of Class $\mathcal{S}$.

Lemma 1 For problem $T P 2|v=1, c \geq n| K_{\max }$, the search for an optimal schedule can be limited to the schedules in which the first move of the transporter from $M_{1}$ to $M_{2}$ starts at time zero.

Proof: If in a feasible schedule $S$ each job is processed on one machine only (i.e., $Q(S)=\varnothing$ ), then without increasing the value of $K_{\max }$ schedule $S$ can be transformed into another schedule in which (i) all jobs to be processed on machine $M_{2}$ are brought there by the first move of the transporter and (ii) that move starts at time zero.

Now, for schedule $S$, suppose that $Q(S) \neq \varnothing$ and that the first move of the transporter starts at time $t_{1}$. Let $N\left(t_{1}\right)$ be the set of jobs such that for each job $j \in N\left(t_{1}\right)$ either its full processing on machine $M_{1}$ or the processing of its portion on $M_{1}$ is completed before time $t_{1}$. In set $N\left(t_{1}\right)$, distinguish between the jobs of set $W_{1}(S)$ and those of set $Q(S)$ by defining 
$W\left(t_{1}\right):=W_{1}(S) \cap N\left(t_{1}\right)$ and $Q\left(t_{1}\right):=Q(S) \cap N\left(t_{1}\right)$. In turn, set $Q\left(t_{1}\right)$ can be partitioned as $Q\left(t_{1}\right)=Q^{\prime}\left(t_{1}\right) \cup Q^{\prime \prime}\left(t_{1}\right)$, where set $Q^{\prime}\left(t_{1}\right)$ consists of all jobs of $Q\left(t_{1}\right)$ that are transported to $M_{2}$ at time $t_{1}$ by the first move of the transporter. Denote the duration of the processing of job $j \in Q^{\prime}\left(t_{1}\right)$ on machine $M_{1}$ before time $t_{1}$ by $x_{j}^{\prime}$. Since the jobs of set $W\left(t_{1}\right)$ do not have to be moved between the machines and the jobs of set $Q^{\prime \prime}\left(t_{1}\right)$ are not moved to $M_{2}$ at time $t_{1}$, it follows that, without increasing the length of the schedule, we can

(i) interchange the jobs and portions of jobs in such a way that machine $M_{1}$ first processes the jobs of set $Q^{\prime}\left(t_{1}\right)$ (each job as one portion), followed by the remaining jobs (or portions of jobs) of set $Q\left(t_{1}\right)$;

(ii) start the first move of the transporter at time $x^{\prime}\left(Q^{\prime}\left(t_{1}\right)\right)$, on completion of all portions of jobs of set $Q^{\prime}\left(t_{1}\right)$ on $M_{1}$.

In the resulting schedule $S^{\prime}$, the processing on machine $M_{2}$ starts upon arrival of the transporter at time $x^{\prime}\left(Q^{\prime}\left(t_{1}\right)\right)+\tau$. Let $k$ be a job of set $Q^{\prime}\left(t_{1}\right)$ whose portion completes on machine $M_{1}$ at time $x^{\prime}\left(Q^{\prime}\left(t_{1}\right)\right)$ in schedule $S^{\prime}$. Without changing the length of the schedule $K_{\max }\left(S^{\prime}\right)$, we can start the first move of the transporter at time $x^{\prime}\left(Q^{\prime}\left(t_{1}\right)\right)-x_{k}^{\prime}$ and move the processing of the portion of job $k$ to machine $M_{2}$ to start at time $x^{\prime}\left(Q^{\prime}\left(t_{1}\right)\right)-x_{k}^{\prime}+\tau$, so that the processing of the other jobs and portions of jobs on $M_{2}$ can still start at time $x^{\prime}\left(Q^{\prime}\left(t_{1}\right)\right)+\tau$. Repeating this process, we obtain a schedule $S^{\prime \prime}$ such that $K_{\max }\left(S^{\prime \prime}\right)=K_{\max }\left(S^{\prime}\right) \leq K_{\max }(S)$ and in $S^{\prime \prime}$ the first move of the transporter starts at time zero.

Lemma 2 For problem $T P 2|v=1, c \geq n| K_{\max }$, the search for an optimal schedule can be limited to the schedules in which the last move of the transporter does not deliver to a machine $M \in\left\{M_{1}, M_{2}\right\}$ the jobs of set $Q(S)$ which still have to be processed on $M$.

Proof: We present the proof for the case that the last move of the transporter is made from machine $M_{2}$ to machine $M_{1}$, i.e., the initial schedule $S$ is a Type 2 schedule; the case of schedule $S$ being a Type 1 schedule is symmetric.

Suppose that in $S$ the transporter starts its last move at time $t$ to arrive at $M_{1}$ at time $t+\tau$. Assume that among the jobs brought by this move, there is a non-empty set $Q^{\prime} \subseteq Q(S)$ of fractional jobs, each of which still has to be processed on $M_{1}$. Denote the start time of the first job (or a portion of a job) on machine $M_{1}$ after time $t+\tau$ by $t^{0}$ and denote the duration of the processing of job $j \in Q^{\prime}$ on machine $M_{1}$ after time $t^{0}$ by $x_{j}^{\prime}$. Interchange the jobs and portions of jobs processed on $M_{1}$ after time $t^{0}$ in such a way that machine $M_{1}$ starting at time $t^{0}$ processes the jobs of set $Q^{\prime}$ (each job as one portion), followed by the remaining jobs, if any. Call the resulting schedule $S^{\prime}$. Let $l$ be the job of set $Q^{\prime}$ that starts on machine $M_{1}$ at time $t^{0}$. Without changing the length of the schedule, we can start the last move of the transporter at time $t^{0}+x_{l}^{\prime}-\tau$ and move the processing of the portion of job $l$ to machine $M_{2}$ to start at time $t^{0}-\tau$, so that the processing of the other jobs and portions of jobs on $M_{1}$ can still start at time $t^{0}+x_{l}^{\prime}$. Repeating this process, we obtain a schedule $S^{\prime \prime}$ such that $K_{\max }\left(S^{\prime \prime}\right) \leq K_{\max }\left(S^{\prime}\right)=K_{\max }(S)$ and in $S^{\prime \prime}$ the last move of the transporter does not deliver any jobs of set $Q(S)$ that need further processing on $M_{1}$.

We now derive several lower bounds on the length of a feasible schedule.

Define

$$
T=\frac{p(N)}{2}+\tau .
$$


Lemma 3 For any schedule $S$ that is feasible for problem $T P 2|v=1, c \geq n| K_{\max }$, the lower bound

$$
K_{\max }\left(S^{*}\right) \geq T
$$

holds.

Proof: For schedule $S$, let $X$ denote the total load on machine $M_{1}$, i.e., the sum of the processing times of the jobs and portions of jobs processed on $M_{1}$. If $S$ is a Type 1 schedule then $K_{\max }\left(S^{*}\right) \geq X+\tau$ and $K_{\max }\left(S^{*}\right) \geq p(N)-X+\tau$. Otherwise, if $S$ is a Type 2 schedule then $K_{\max }\left(S^{*}\right) \geq X$ and $K_{\max }\left(S^{*}\right) \geq p(N)-X+2 \tau$. In either case, the required lower bound (2) follows immediately.

It is not possible that there is exactly one move in an optimal schedule, since the length of such a schedule would be equal to $p(N)+\tau$, which is larger than $p(N)$, the length of a Type 0 schedule. Thus, in each Type 1 schedule there are at least three moves, so that the first move and the last move of the transporter are made from the top machine $M_{1}$, and the schedule terminates when all completed jobs are collected on board of the transporter at the bottom machine $M_{2}$. For any Type 1 schedule $S$ the lower bound

$$
K_{\max }(S) \geq p_{j}+\tau
$$

holds for each job $j \in N$. Indeed, if a job $p_{j}$ is completed on the top machine $M_{1}$, it has to be moved to machine $M_{2}$, where the schedule terminates. If it is completed on machine $M_{2}$, it has to be brought there before its (possibly, partial) processing may start on that machine. This implies the bound (3).

In each Type 2 schedule the first move of the transporter is made from the top machine $M_{1}$, the last move is made from the bottom machine $M_{2}$, and the schedule terminates when all completed jobs are collected on board of the transporter at the top machine $M_{1}$.

It is clear that for any Type 2 schedule $S$ a lower bound

$$
K_{\max }(S) \geq p_{j}
$$

holds for each job $j \in N$. Besides, if in a Type 2 schedule $S$ job $j$ or its part is processed on machine $M_{2}$ then

$$
K_{\max }\left(S^{*}\right) \geq p_{j}+2 \tau .
$$

To see this, notice that job $j$ must be brought to $M_{2}$ and returned to $M_{1}$, and while it is processed on $M_{2}$ or being moved it cannot be processed on $M_{1}$.

Definition $2 A$ job $j \in N$ is said to have rank $r, r \in\{0,1,2,3\}$ if

$$
p_{j}+r \tau \geq T \text {. }
$$

It is clear that a job of rank $r-1$ is also a job of rank $r, r \in\{1,2,3\}$. The rank of a job plays an important role in the proof of the main statement of this section, Theorem 1 , and in several auxiliary statements.

In the proofs and algorithms presented in this paper, we will often use the following splitting procedure.

\section{Procedure $\operatorname{Split}(X, Y, \gamma)$}

Input: Two sets $X$ and $Y=\{\sigma(1), \ldots, \sigma(y)\}$ of jobs and a bound $\gamma$ such that $|X| \geq 0$, $|Y|=y>1$, and $p(X)<\gamma, p(X)+p(Y)>\gamma$ 


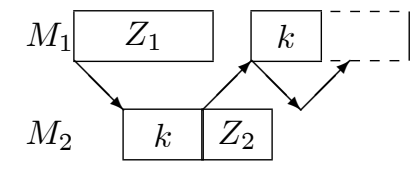

(a)

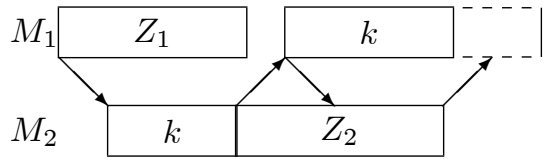

(b)

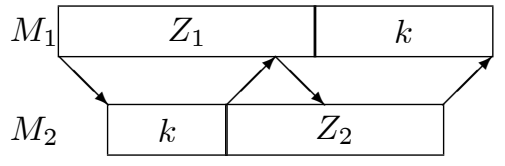

$(c)$

Figure 1: A preemptive schedule $S_{4}(k)$ with four moves

Output: A set $Y^{\prime} \subset Y$ and a job $u \in Y$ that is split in two portions $p_{u}=x_{u}+y_{u}$, so that $p(X)+p\left(Y^{\prime}\right)+x_{u}=\gamma$

Step 1: Considering the jobs of set $Y$ in the order given by the list $\sigma$, find the position $v$, where $1 \leq v \leq y$ such that

$$
p(X)+\sum_{j=1}^{v-1} p_{\sigma(j)}<\gamma, p(X)+\sum_{j=1}^{v} p_{\sigma(j)} \geq \gamma .
$$

Step 2: Output $Y^{\prime}:=\{\{\sigma(1), \ldots, \sigma(v-1)\}\}, u:=\sigma(v), x_{u}:=\gamma-p(X)-p\left(Y^{\prime}\right), y_{u}:=p_{u}-x_{u}$.

It is clear that the running time of Procedure Split is linear in $|X \cup Y|$.

Given a schedule $S$, the set of whole jobs that are processed on machine $M_{1}$ (or on machine $M_{2}$ only) is denoted by $Z_{1}(S)\left(Z_{2}(S)\right.$, respectively). If no confusion arises, we may drop the reference to a schedule and write $Z_{1}$ and $Z_{2}$.

Lemma 4 Let $A$ and $B$ be disjoint sets of jobs such that $p(A) \leq T$ and $p(A)+p(B)>T$. Suppose that Procedure $\operatorname{Split}(A, B, T)$ is run and a set $B^{\prime} \subset B$ and a job $k \in B \backslash B^{\prime}$ are found such that $p(A)+p\left(B^{\prime}\right)+x_{k}=T$, where $0<x_{k}<p_{k}$. Define $Z_{1}:=A \cup B^{\prime}$ and $Z_{2}:=N \backslash\left(Z_{1} \cup\{k\}\right)$. Let $S_{4}(k)$ be a Type 2 schedule shown in Figure 1. Then either

- job $k$ is a rank 2 job,

- or $K_{\max }\left(S_{4}(k)\right)=T$, provided that $p\left(Z_{2}\right) \geq 2 \tau$.

Proof: In schedule $S_{4}(k)$ only job $k$ is fractional, and $p\left(Z_{1}\right)+x_{k}=p\left(Z_{2}\right)+y_{k}+2 \tau=T$. If $y_{k}+2 \tau>T-x_{k}=p\left(Z_{1}\right)$, then $p_{k}+2 \tau>T$, i.e., job $k$ is a rank 2 job, and the structure of schedule $S_{4}(k)$ is as shown in Figure 1(a) or (b). If $y_{k}+2 \tau \leq T-x_{k}=p\left(Z_{1}\right)$ then job $k$ is delivered to machine $M_{1}$ before all jobs of set $Z_{1}$ are completed on that machine. Furthermore, due to the condition $p\left(Z_{2}\right) \geq 2 \tau$, the next downward move of the transporter is finished no later than all jobs are completed on machine $M_{2}$. Thus, the structure of schedule $S_{4}(k)$ is as shown in Figure $1(\mathrm{c})$ and $K_{\max }\left(S_{4}(k)\right)=T$.

Lemma 5 Let $A$ and $B$ be disjoint sets of jobs such that $p(A) \leq T-\tau$ and $p(A)+p(B)>T-\tau$. Suppose that Procedure $\operatorname{Split}(A, B, T-\tau)$ is run and a set $B^{\prime} \subset B$ and a job $k \in B \backslash B^{\prime}$ are found such that $p(A)+p\left(B^{\prime}\right)+x_{k}=T-\tau$, where $0<x_{k}<p_{k}$. Define $Z_{1}:=A \cup B^{\prime}$ and $Z_{2}:=N \backslash\left(Z_{1} \cup\{k\}\right)$. Let $S_{3}(k)$ be a Type 1 schedule shown in Figure 2. Then either 


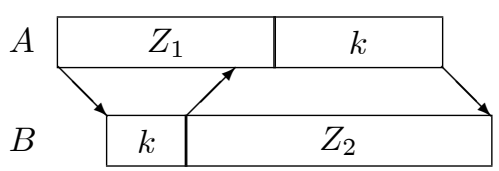

(a)

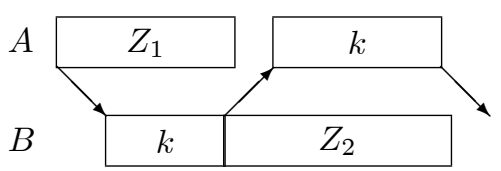

(b)

Figure 2: A preemptive schedule $S_{3}(k)$ with three moves

- $K_{\max }\left(S_{3}(k)\right)=T$

- or job $k$ is a rank 3 job and $K_{\max }\left(S_{3}(k)\right)=p_{k}+3 \tau$.

Proof: In schedule $S_{3}(k)$ only job $k$ is fractional, and the load of each machine is equal to $T-\tau$, i.e., $p\left(Z_{1}\right)+x_{k}=p\left(Z_{2}\right)+y_{k}=T-\tau$. If $y_{k}+2 \tau \leq(T-\tau)-x_{k}=p\left(Z_{1}\right)$ then job $k$ is delivered to machine $M_{1}$ before all jobs of set $Z_{1}$ are completed on that machine, the structure of schedule $S_{3}(k)$ is as shown in Figure $2(\mathrm{a})$, and $K_{\max }\left(S_{3}(k)\right)=T$. If $y_{k}+2 \tau>(T-\tau)-x_{k}$, then $p_{k}+3 \tau>T$, i.e., job $k$ is a rank 3 job. Moreover, job $k$ can only start on $M_{1}$ at time $y_{k}+2 \tau$, so that $K_{\max }\left(S_{3}(k)\right)=p_{k}+3 \tau$ and the structure of schedule $S_{3}(k)$ is as shown in Figure 2(b).

Lemma 6 For problem $T P 2|v=1, c \geq n| K_{\max }$, suppose that $S$ is a schedule with at least five moves. If for some job $j \in N$ the inequality

$$
p_{j}+3 \tau>K_{\max }(S)
$$

holds, then

$$
p_{j}>2 \tau
$$

and

$$
p_{j}>p(N) / 4 \text {. }
$$

Proof: Since there are at least five moves in schedule $S$, it follows that a lower bound

$$
K_{\max }(S) \geq 5 \tau
$$

holds. Then (7) implies (8). Due to (2), we deduce from (7) that $p_{j}+3 \tau>T=p(N) / 2+\tau$, which together with (8) yields (9).

Theorem 1 For problem $T P 2|v=1, c \geq n| K_{\max }$, the search for an optimal schedule can be limited to schedules with at most 4 moves of the transporter.

Proof: Suppose that $S^{0}$ is an optimal schedule with at least five moves, and there is no optimal schedule with less than five moves. For schedule $S=S^{0}$ inequality (10) holds. To prove the theorem, we will show that we can always construct a schedule $S^{*}$ with 2,3 or 4 moves and such that $K_{\max }\left(S^{*}\right) \leq K_{\max }\left(S^{0}\right)$.

If necessary, renumber the jobs in such a way that

$$
p_{1} \geq p_{2} \geq p_{3} \geq \max \left\{p_{j} \mid j \in N \backslash\{1,2,3\}\right\} .
$$




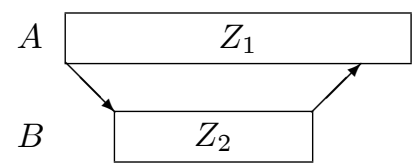

(a)

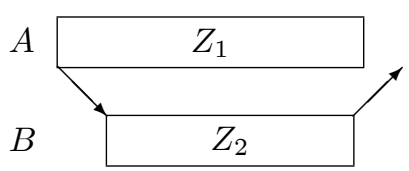

(b)

Figure 3: A schedule with two moves

Rank 0 job. Assume that job 1 has rank 0 . Construct the following schedule $S_{2}(1)$ with two moves, in which machine $M_{1}$ processes job 1 alone and the rest of the jobs are processed on machine $M_{2}$. The first move of the transporter (from $M_{1}$ to $M_{2}$ ) starts at time zero, and the second move (from $M_{2}$ to $M_{1}$ ) starts at $\tau+p(N)-p_{1}$ to complete at $2 \tau+p(N)-p_{1}$. See Figure 3(a) with $Z_{1}=\{1\}, Z_{2}=N \backslash\{1\}$. We use (6) with $r=0$ to deduce $2 \tau+p(N)-p_{1} \leq 2 T-T=T \leq p_{1}$, so that $K_{\max }\left(S_{2}(1)\right)=p_{1}$, and this schedule is optimal due to (4).

In the rest of this proof, it is assumed that there is no job of rank 0 .

Rank 1 job. Suppose that job 1 has rank 1 , which is equivalent to $p_{1} \geq p(N) / 2$, i.e., there are no other rank 1 jobs.

Construct a Type 1 schedule $S_{3}(1)$ in which machine $M_{1}$ processes only job 1 while machine $M_{2}$ processes the rest of the jobs. At time zero, the transporter leaves job 1 on machine $M_{1}$, moves to $M_{2}$ to deliver the jobs of set $N \backslash\{1\}$, immediately returns to machine $M_{1}$, and then moves the completed job 1 from $M_{1}$ to $M_{2}$.

If $K_{\max }\left(S_{3}(1)\right)=3 \tau$, which happens if $p_{1}<2 \tau$, then $K_{\max }\left(S_{3}(1)\right)<5 \tau \leq K_{\max }\left(S^{0}\right)$. Otherwise, since $p_{1} \geq p(N) / 2 \geq p(N)-p_{1}$, it follows that $K_{\max }\left(S_{3}(1)\right)=p_{1}+\tau$. If there exists an optimal Type 1 schedule then $S_{3}(1)$ is optimal due to (3).

Thus, in our further analysis of the rank 1 job, we assume that an optimal schedule $S^{0}$ is a Type 2 schedule, i.e., in this schedule there are at least 6 moves, and therefore $K_{\max }\left(S^{0}\right) \geq 6 \tau$ and additionally $K_{\max }\left(S^{0}\right)<K_{\max }\left(S_{3}(1)\right)=p_{1}+\tau$. Notice that this implies that in schedule $S^{0}$ job 1 is fully processed on machine $M_{2}$, since otherwise we would have $K_{\max }\left(S^{0}\right) \geq p_{1}+2 \tau$ due to $(5)$.

Consider the situation that

$$
p_{2}+2 \tau<T
$$

Run Procedure Split $(\{1\}, N \backslash\{1\}, T)$ to find a set $H_{1} \subseteq N \backslash\{1\}$ and a job $k$ such that $p_{1}+p\left(H_{1}\right)+x_{k}=T$. If $x_{k}=0$, then we create a schedule with two moves in which machine $M_{1}$ processes the jobs of set $H_{1} \cup\{1\}$, while machine $M_{2}$ processes the remaining jobs; the length of this schedule is $T$; see Figure 3 with $Z_{1}=H_{1} \cup\{1\}, Z_{2}=N \backslash Z_{1}$. If $0<x_{k}<p_{k}$, then notice that $p_{k}+2 \tau \leq p_{2}+2 \tau<T$. If for set $Z_{2}=N \backslash\left(H_{1} \cup\{1, k\}\right)$, the inequality $p\left(Z_{2}\right) \geq 2 \tau$ holds, then due to Lemma 4 applied to $A=\{1\}$ and $B=N \backslash\{1\}$, the structure of schedule $S_{4}(k)$ with four moves is as shown in Figure $1(\mathrm{c})$, and $K_{\max }\left(S_{4}(k)\right)=T$, so that this schedule is optimal due to (2). On the other hand, if $p\left(Z_{2}\right)<2 \tau$, then we derive $p_{1}+p\left(H_{1}\right)+p\left(Z_{2}\right)=$ $p(N)-p_{k}>T$, and since $p_{1}+p\left(H_{1}\right)<T$, we can run Procedure $\operatorname{Split}\left(\{1\} \cup H_{1}, Z_{2}, T\right)$ to find a set $H_{1}^{\prime} \subseteq Z_{2}$ and job $h \in Z_{2}$ such that $p_{1}+p\left(H_{1}\right)+p\left(H_{1}^{\prime}\right)+x_{h}=T$. If $x_{h}=0$, then we create a schedule with two moves in which machine $M_{1}$ processes the jobs of set $H_{1} \cup H_{1}^{\prime} \cup\{1\}$, while machine $M_{2}$ processes the remaining jobs; the length of this schedule is $T$, see Figure 3 with $Z_{1}=H_{1} \cup H_{1}^{\prime} \cup\{1\}, Z_{2}=N \backslash Z_{1}$. If $0<x_{h}<p_{h}$, then notice that $p_{h}+2 \tau \leq p_{2}+2 \tau<T$. 
Define $Z_{1}^{\prime}:=H_{1} \cup H_{1}^{\prime} \cup\{1\}$ and $Z_{2}^{\prime}:=N \backslash\left(Z_{1}^{\prime} \cup\{h\}\right)$. If the inequality $p\left(Z_{2}^{\prime}\right) \geq 2 \tau$ holds, then due to Lemma 4 applied to $A=\{1\} \cup H_{1}$ and $B=Z_{2}$, the structure of schedule $S_{4}(h)$ with four moves is as shown in Figure $1(\mathrm{c})$ with $k=h$, so that $K_{\max }\left(S_{4}(h)\right)=T$ and this schedule is optimal due to (2). On the other hand, if $p\left(Z_{2}^{\prime}\right)<2 \tau$, then $K_{\max }\left(S_{4}(h)\right)=\tau+y_{h}+3 \tau$ and since $y_{h}<p\left(Z_{2}\right)<2 \tau$, we conclude that $K_{\max }\left(S_{4}(h)\right)=4 \tau+y_{h}<6 \tau \leq K_{\max }\left(S^{0}\right)$.

Assume now that

$$
p_{2}+2 \tau \geq T
$$

i.e., job 2 is a rank 2 job. Notice that $p(N)-p_{2}=2 T-2 \tau-p_{2} \leq T$.

If in schedule $S^{0}$ job 2 or its part is processed on machine $M_{2}$, then (5) holds for $j=2$. We can create a schedule $S_{2}(2)$ with two moves, in which machine $M_{2}$ processes job 2 alone and the rest of the jobs are processed on machine $M_{1}$; see Figure 3 with $Z_{1}=N \backslash\{2\}$, $Z_{2}=\{2\}$. The first move of the transporter (from $M_{1}$ to $M_{2}$ ) starts at time zero, and the second move (from $M_{2}$ to $M_{1}$ ) starts at $\tau+p_{2}$ to complete at $2 \tau+p_{2} \geq p(N)-p_{2}$. Thus, $K_{\max }\left(S_{2}(2)\right)=p_{2}+2 \tau$, and this schedule is optimal due to (5) with $j=2$.

If in schedule $S^{0}$ job 2 is fully processed on machine $M_{1}$, then $K_{\max }\left(S^{0}\right) \geq p_{1}+p_{2}$. However, $K_{\max }\left(S^{0}\right)<p_{1}+\tau$ implies that $p_{2}<\tau$, which together with $p_{2}+2 \tau \geq T$ yields $3 \tau>T$. We use $K_{\max }\left(S^{0}\right) \geq 6 \tau$ to derive $K_{\max }\left(S^{0}\right)>2 T>p(N)$, i.e., $S^{0}$ is worse than a Type 0 schedule, which is not possible.

No Rank 1 jobs. From now on, we assume that there is no job of rank 1, so that $p_{j}<T-\tau$ for each $j \in N$.

In the remaining part of this proof, a job $j \in N$ is called special, if (i) job $j$ is a rank 3 job, and (ii) $p_{j}+3 \tau>K_{\max }\left(S^{0}\right)$. Let $g$ denote the number of special jobs. As follows from Lemma 6 , for each special job $j$ the inequalities (8) and (9) hold, so that $g \in\{0,1,2,3\}$.

If $g \in\{0,1\}$, then run Procedure Split $(\{1\}, N \backslash\{1\}, T-\tau)$ to find a set $H_{1} \subset N \backslash\{1\}$ and a job $k \in N \backslash\{1\}$ such that $p_{1}+p\left(H_{1}\right)+x_{k}=T-\tau$. If $x_{k}=0$, we construct a non-preemptive schedule with three moves of length $T$, in which machine $M_{1}$ processes the set of jobs $H_{1} \cup\{1\}$, and machine $M_{2}$ processes the rest of the jobs. If $x_{k}>0$, then notice that job $k$ is not special since $p_{k} \leq p_{1}$, i.e., either job $k$ is not a rank 3 job and $p_{k}+3 \tau<T$, or it is a rank 3 job but $p_{k}+3 \tau \leq K_{\max }\left(S^{0}\right)$. Applying Lemma 5 with $A=\{1\}$ and $B=N \backslash\{1\}$, we derive that for schedule $S_{3}(k)$ either $K_{\max }\left(S_{3}(k)\right)=T$ or $K_{\max }\left(S_{3}(k)\right)=p_{k}+3 \tau \leq K_{\max }\left(S^{0}\right)$ holds.

If $g=2$, then we have two special jobs, job 1 and job 2. Run Procedure $\operatorname{Split}(\{1\}, N \backslash\{1,2\}, T-\tau)$ to find a set $H_{1} \subset N \backslash\{1,2\}$ and a job $k \in N \backslash\{1,2\}$ such that $p_{1}+p\left(H_{1}\right)+x_{k}=T-\tau$. As above, if $x_{k}=0$, we construct a non-preemptive schedule with three moves of length $T$. If $x_{k}>0$, notice that job $k$ is not special since $p_{k}<p_{2} \leq p_{1}$, i.e., job $k$ either is not a rank 3 job and $p_{k}+3 \tau<T$, or it is a rank 3 job but $p_{k}+3 \tau \leq K_{\max }\left(S^{0}\right)$. Applying Lemma 5 with $A=\{1\}$ and $B=N \backslash\{1,2\}$, we derive that a Type 1 schedule $S_{3}(k)$ is of structure shown in Figure $2(\mathrm{a})$ and $K_{\max }\left(S_{3}(k)\right)=T$.

Finally, assume that $g=3$, i.e., we have three special jobs: job 1 , job 2 and job 3 . Let $R$ denote the set of all other jobs; none of these jobs is special.

We prove that in the case under consideration job 2 cannot have rank 2. Assuming the opposite, we derive

$$
p_{2}+2 \tau \geq T=\frac{p(N)}{2}+\tau \geq \frac{p_{1}}{2}+\frac{p_{2}}{2}+\frac{p_{3}}{2}+\tau,
$$

which reduces to

$$
\frac{p_{2}}{2}+\tau \geq \frac{p_{1}}{2}+\frac{p_{3}}{2}
$$


However the latter inequality is impossible due to $p_{1} \geq p_{2}$ and (8) with $j=3$.

Thus, job 2 is not a rank 2 job, i.e., $p_{2}+2 \tau<T$. It follows that $p_{1}+p_{3}+p(R)=$ $(2 T-2 \tau)-p_{2}>T$. On the other hand, $p_{1}+p(R)=p(N)-p_{2}-p_{3}<p(N)-p(N) / 4-$ $p(N) / 4=p(N) / 2<T$. Run Procedure $\operatorname{Split}(\{1, R\},\{3\}, T)$ to find the value $x_{3}$ such that $p_{1}+p(R)+x_{3}=T$. Notice that job 3 is not a rank 2 job since $p_{3} \leq p_{2}<T-2 \tau$. Applying Lemma 4 with $A=\{1, R\}, B=\{3\}$ and $k=3$, we derive that a Type 2 schedule $S_{4}(3)$ with four moves is of structure shown in Figure $1(\mathrm{c})$ and $K_{\max }\left(S_{4}(3)\right)=T$.

This completes the proof of the theorem.

An immediate implication of Lemmas 1 and 2 and of Theorem 1 is that an optimal schedule will be sought for among the schedules of Class $\mathcal{S}$ with two, three or four moves.

Lemma 7 For problem $T P 2|v=1, c \geq n| K_{\max }$, the search for the best schedule with two moves can be limited to the non-preemptive schedules.

Proof: Suppose for a schedule $S \in \mathcal{S}$ the set $Q(S)$ of fractional jobs is not empty. By Lemma 1 each job $j \in Q(S)$ is brought to machine $M_{2}$ by the first move of the transporter that starts at time zero, and has to be completed subsequently on $M_{1}$. However, by Lemma 2 , the second (last) move of the transporter cannot deliver any unfinished jobs.

Lemma 8 For problem $T P 2|v=1, c \geq n| K_{\max }$, let $S_{3} \in \mathcal{S}$ be a schedule with three moves in which some jobs are fractional. Then without increasing the value of the objective function, schedule $S_{3}$ can be transformed into either a non-preemptive schedule or into a schedule $S_{3}(k)$ in which only some job $k \in Q\left(S_{3}\right)$ remains fractional. In the latter case, the lower bound

$$
K_{\max }\left(S_{3}(k)\right) \geq p_{k}+3 \tau
$$

holds.

Proof: Suppose that in schedule $S_{3} \in \mathcal{S}$ a job $j \in Q\left(S_{3}\right)$ starts its processing on machine $M_{1}$. Since the first move of the transporter starts at time zero, it follows that job $j$ is brought to machine $M_{2}$ by the third (last) move. However, upon arrival at $M_{2}$ job $j$ requires further processing, which contradicts Lemma 2.

Thus, any job of set $Q\left(S_{3}\right)$ starts its processing on machine $M_{2}$ and is delivered there at time $\tau$. After their processing on machine $M_{2}$ is finished, all these jobs together are delivered to $M_{1}$ to be completed on that machine.

Suppose that $\left|Q\left(S_{3}\right)\right|=\ell \geq 2$, and renumber (if required) the jobs of this set so that $Q\left(S_{3}\right)=\{1,2, \ldots, \ell\}$. Without increasing the objective function, we can transform schedule $S_{3}$ into a new schedule $S_{3}^{\prime}$ in which:

- the jobs of set $Q\left(S_{3}\right)$ are processed on machine $M_{2}$ in the time interval $\left[\tau, \tau+y\left(Q\left(S_{3}\right)\right)\right]$ and are followed by an arbitrary sequence of the whole jobs;

- the second move of the transporter (from $M_{2}$ to $M_{1}$ ) starts immediately after all portions of the fractional jobs are completed on $M_{2}$, i.e., at time $\tau+y\left(Q\left(S_{3}\right)\right)$;

- the jobs of set $Q\left(S_{3}\right)$ are processed on machine $M_{1}$ as a block after all whole jobs on that machine. 


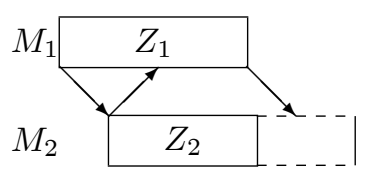

(a)

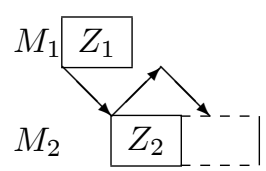

(b)

Figure 4: A non-preemptive schedule with three moves

Thus, in schedule $S_{3}^{\prime}$, the set of jobs is partitioned into the set of fractional jobs $Q\left(S_{3}^{\prime}\right)=$ $Q\left(S_{3}\right)$ and the sets $Z_{1}\left(S_{3}^{\prime}\right)$ and $Z_{2}\left(S_{3}^{\prime}\right)$ of the whole jobs assigned to machine $M_{1}$ and machine $M_{2}$, respectively. It follows that

$$
\begin{aligned}
K_{\max }\left(S_{3}\right) \geq & K_{\max }\left(S_{3}^{\prime}\right)=\max \left\{p\left(Z_{1}\left(S_{3}^{\prime}\right)\right)+x\left(Q\left(S_{3}\right)\right)+\tau, \tau+y\left(Q\left(S_{3}\right)\right)+p\left(Z_{2}\left(S_{3}^{\prime}\right)\right),\right. \\
& \left.\tau+y\left(Q\left(S_{3}\right)\right)+\tau+x\left(Q\left(S_{3}\right)\right)+\tau\right\}
\end{aligned}
$$

Run Procedure Split $\left(\varnothing, Q\left(S_{3}\right), y\left(Q\left(S_{3}\right)\right)\right.$, which will scan the jobs of set $Q\left(S_{3}\right)$ in the order of their numbering and find a job $k \leq \ell$ such that

$$
\sum_{j=1}^{k-1} p_{j}<y\left(Q\left(S_{3}\right)\right), \sum_{j=1}^{k} p_{j} \geq y\left(Q\left(S_{3}\right)\right) .
$$

If $\sum_{j=1}^{k} p_{j}=y\left(Q\left(S_{3}\right)\right)$ then schedule $S_{3}^{\prime}$ can be transformed into a non-preemptive schedule $S_{3}^{\prime \prime}$ shown in Figure 4 with $Z_{1}=Z_{1}\left(S_{3}^{\prime}\right) \cup\{k+1, \ldots, \ell\}$ and $Z_{2}=Z_{2}\left(S_{3}^{\prime}\right) \cup\{1, \ldots, k\}$. It is clear that

$$
K_{\max }\left(S_{3}^{\prime \prime}\right)=\max \left\{p\left(Z_{1}\left(S_{3}^{\prime}\right)\right)+x\left(Q\left(S_{3}\right)\right)+\tau, \tau+y\left(Q\left(S_{3}\right)\right)+p\left(Z_{2}\left(S_{3}^{\prime}\right)\right), 3 \tau\right\} \leq K_{\max }\left(S_{3}^{\prime}\right) .
$$

Alternatively, create a schedule $S_{3}(k)$ shown in Figure 2 with $Z_{1}=Z_{1}\left(S_{3}^{\prime}\right) \cup\{k+1, \ldots, \ell\}$ and $Z_{2}=Z_{2}\left(S_{3}^{\prime}\right) \cup\{1, \ldots, k-1\}$ and the fractional job $k$ split by $y_{k}=y\left(Q_{3}\left(S_{3}\right)\right)-\sum_{j=1}^{k-1} p_{j}$, $x_{k}=p_{k}-y_{k}$, so that

$$
y\left(Q_{3}\left(S_{3}\right)\right)=y_{k}+\sum_{j=1}^{k-1} p_{j}, x\left(Q_{3}\left(S_{3}\right)\right)=x_{k}+\sum_{j=k+1}^{\ell} p_{j} .
$$

Thus, we obtain that

$$
\begin{aligned}
K_{\max }\left(S_{3}\right) \geq & K_{\max }\left(S_{3}(k)\right)=\max \left\{p\left(Z_{1}\left(S_{3}^{\prime}\right)\right)+x\left(Q\left(S_{3}\right)\right)+\tau, \tau+y\left(Q\left(S_{3}\right)\right)+p\left(Z_{2}\left(S_{3}^{\prime}\right)\right),\right. \\
& \left.\tau+y_{k}+\tau+x_{k}+\tau\right\}
\end{aligned}
$$

which in particular implies the required lower bound (12).

Lemma 9 For problem $T P 2|v=1, c \geq n| K_{\max }$, the search for the best schedule with four moves can be limited to the schedules in which some jobs are assigned to be processed preemptively on both machines.

Proof: Suppose $S_{4}^{*}$ is the best schedule with four moves in which no job is processed on both machines. Let $Z_{1}$ and $Z_{2}$ denote the sets of jobs assigned to machine $M_{1}$ and to machine $M_{2}$, respectively. It follows that $K_{\max }\left(S_{4}^{*}\right) \geq \max \left\{p\left(Z_{1}\right), p\left(Z_{2}\right)+2 \tau\right\}$. But there exists a two-move schedule of length $\max \left\{p\left(Z_{1}\right), p\left(Z_{2}\right)+2 \tau\right\}$, as shown in Figure 3 . 
Lemma 10 For problem $T P 2|v=1, c \geq n| K_{\max }$, let $S_{4}$ be a schedule with four moves. Then

$$
K_{\max }\left(S_{4}\right) \geq 4 \tau+y(Q)
$$

where $Q$ is the set of jobs that are processed with preemption on both machines.

Proof: In schedule $S_{4} \in \mathcal{S}$, any job of set $Q=Q\left(S_{4}\right)$ is delivered to $M_{2}$ either by the first move of the transporter (and each of these jobs is brought back to be completed on $M_{1}$ by the second move) or by the third move of the transporter (and each of these jobs is completed on $M_{2}$ before it is brought back to $M_{1}$ by the last move of the transporter). This implies that no job of set $Q$ is processed on machine $M_{2}$ between the second and the third moves of the transporter. The desired lower bound (13) follows immediately.

Theorem 2 For problem $T P 2|v=1, c \geq n| K_{\max }$, let $W_{2}^{*}$ be a set of jobs such that $p\left(W_{2}^{*}\right) \leq$ $T-2 \tau$ and $p\left(W_{2}^{*}\right) \geq p(W)$ for any set $W \subseteq N$ with $p(W) \leq T-2 \tau$. Let $S_{4}^{*}$ be the best schedule with four moves. Then

$$
K_{\max }\left(S_{4}^{*}\right) \geq y^{*}+4 \tau
$$

where

$$
y^{*}=\frac{p(N)-p\left(W_{2}^{*}\right)}{2}-2 \tau
$$

Proof: Suppose that in schedule $S_{4}^{*}$ machine $M_{2}$ processes a set $W_{2}$ of jobs that are processed on this machine only and a set $Q$ of the jobs that are processed preemptively on both machines.

It is clear that the length of schedule $S_{4}^{*}$ cannot be shorter than the total processing of the jobs assigned to machine $M_{1}$, i.e.,

$$
K_{\max }\left(S_{4}^{*}\right) \geq p(N)-p\left(W_{2}\right)-y(Q),
$$

Due to Lemma 10, we have that

$$
K_{\max }\left(S_{4}^{*}\right) \geq y(Q)+4 \tau .
$$

For a fixed set $W_{2}$, let $y$ be the root of the equation

$$
p(N)-p\left(W_{2}\right)-y(Q)=y(Q)+4 \tau,
$$

i.e.,

$$
y=\frac{p(N)-p\left(W_{2}\right)}{2}-2 \tau .
$$

Then $K_{\max }\left(S_{4}^{*}\right) \geq y+4 \tau$, and by definition of set $W_{2}^{*}$ the lower bound (14) holds.

The examples below exhibit instances of problem $T P 2|v=1, c \geq n| K_{\max }$ for which an optimal schedule includes two, three or four moves of the transporter. The examples also demonstrate that all established lower bounds are tight.

In all five listed instances the transportation time $\tau$ is equal to 4 . Optimal schedules for Instances 1 and 2 have no preemption; in the other schedules exactly one job is fractional. For Instances 1-4 the value of $T$ is a tight lower bound, possibly together with another bound. For Instance 5 , the optimal length of the schedule is strictly larger than $T$. Here $T=16$, so that either $W_{2}^{*}=\{2\}$ or $W_{2}^{*}=\{3\}$ (as in Figure $7(\mathrm{~b})$ ), and $p\left(W_{2}^{*}\right)=7<T-2 \tau=8$, see Theorem 2. Thus, the value of $y^{*}=0.5$ is computed in accordance with (15). 


\begin{tabular}{|c|c|c|c|c|c|c|c|c|}
\hline Instance & $p_{1}$ & $p_{2}$ & $p_{3}$ & $T$ & $K_{\max }(S)$ & Tight lower bound & Moves in $S^{*}$ & Chart of $S^{*}$ \\
\hline 1 & 10 & 7 & 5 & 15 & 15 & $(2,5)$ & 2 & Figure 5 \\
\hline 2 & 10 & 7 & 3 & 14 & 14 & $(2)$ & 3 & Figure 6(a) \\
3 & 9 & 2 & 9 & 14 & 14 & $(2,12)$ & 3 & Figure 6(b) \\
\hline 4 & 10 & 8 & 8 & 17 & 17 & $(2)$ & 4 & Figure 7(a) \\
5 & 10 & 7 & 7 & 16 & 16.5 & $(14)$ & 4 & Figure 7(b) \\
\hline
\end{tabular}

Table 1: Instances of the problem

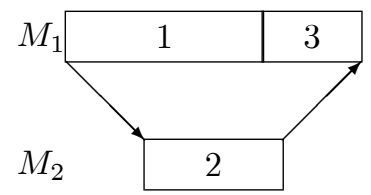

Figure 5: An optimal schedule with two moves for Instance 1

Lemmas 1 and 2, Theorem 1 and the presented examples imply that we can search for an optimal schedule among the schedules of class $\mathcal{S}$, which include either an even (two or four) number of moves or three moves. We split our further consideration accordingly.

In this paper, in various algorithms we use the following statement; see Lemma 4.6.1 in Kellerer et al. (2004).

Theorem 3 Consider the subset-sum problem of the form

$$
\begin{array}{ll}
\max & \sum_{j \in H} p_{j} x_{j} \\
& \sum_{j \in H} p_{j} x_{j} \leq c \\
& x_{j} \in\{0,1\}, j \in H \subseteq N,
\end{array}
$$

This problem admits an FPTAS that for a given positive $\varepsilon$ either finds an optimal solution $x_{j}^{*} \in\{0,1\}, j \in H$ such that

$$
\sum_{j \in H} p_{j} x_{j}^{*}<(1-\varepsilon) c
$$

or finds an approximate solution $x_{j}^{\varepsilon} \in\{0,1\}, j \in H$, such that

$$
(1-\varepsilon) c \leq \sum_{j \in H} p_{j} x_{j}^{\varepsilon} \leq c .
$$

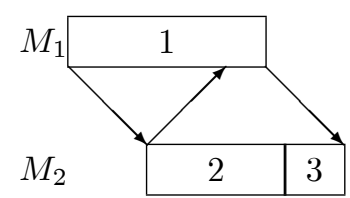

$(a)$

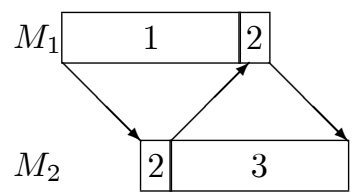

(b)

Figure 6: An optimal schedule with three moves for Instances 2 and 3 


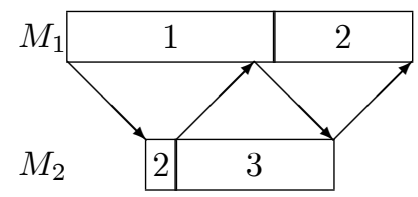

(a)

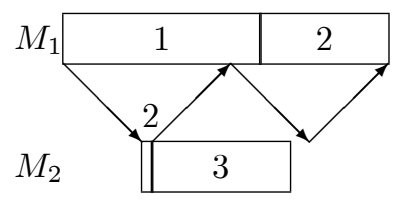

(b)

Figure 7: An optimal schedule with four moves for Instances 4 and 5

Such an FPTAS requires no more than $O(n / \varepsilon)$ time.

\section{Finding the Best Schedule with an Even Number of Moves}

In this section, we show that finding the best schedule with two moves is an NP-hard problem. We also give an algorithm that behaves as an FPTAS, provided that the number of moves in an optimal schedule is either 2 or 4 .

Theorem 4 For problem $T P 2|v=1, c \geq n| K_{\max }$, finding the best schedule with two moves is NP-hard in the ordinary sense.

Proof: To prove the theorem, we provide a reduction of an arbitrary instance of PARTITION to the decision version of problem $T P 2|v=1, c \geq n| K_{\max }$ with $n=r+1$ jobs.

Define $\tau:=2 E$ and $p_{j}:=e_{j}, j=1,2, \ldots, r$, while $p_{r+1}:=4 E$. We show that for the constructed instance a schedule $S$ with two moves such that $K_{\max }(S) \leq 5 E$ exists if and only if PARTition has a solution.

Suppose that PARTition has a solution, and $R_{1}$ and $R_{2}$ are the required subsets with $e\left(R_{1}\right)=e\left(R_{2}\right)=E$. Define a schedule $S$ with two moves of the transporter, in which machine $M_{1}$ processes the jobs of the set $R_{1}$ and job $r+1$ in the time interval [0,5E], while machine $M_{2}$ processes the jobs of set $R_{2}$ in the time interval $[2 E, 3 E]$. The transporter brings the jobs of set $R_{2}$ to machine $M_{2}$ at time $2 E$, waits till the jobs are completed and brings them back to $M_{1}$. It is easy to verify that $K_{\max }(S)=5 E$.

Suppose that schedule $S$ with two moves and of length at most $5 E$ exists. Due to Lemma 7 , there is no fractional job in $S$. Job $r+1$ is processed on machine $M_{1}$; otherwise, $K_{\max }(S) \geq$ $\tau+p_{r+1}+\tau=6 E$. Let the total processing time on machine $M_{2}$ be denoted by $X$. If $X<E$ then $K_{\max }(S) \geq p_{r+1}+(2 E-X)>5 E$, which is impossible. If $X>E$, then $K_{\max }(S) \geq 2 \tau+X>5 E$. Therefore, $X=E$. This implies that the total processing time on machine $M_{1}$ is equal to $5 E$. If for $i \in\{1,2\}$ we denote the subset of jobs of set $\{1,2, \ldots, r\}$ assigned to machine $M_{i}$ by $R_{i}$, then the sets $R_{1}$ and $R_{2}$ form a solution to PARTition. This proves the theorem.

Theorem 5 For problem $T P 2|v=1, c \geq n| K_{\max }$ with $p(N) \geq 4 \tau$, finding the best preemptive schedule with four moves is NP-hard in the ordinary sense.

Proof: As in the previous NP-hardness proof, we reduce PARTITION to the decision version of problem $T P 2|v=1, c \geq n| K_{\max }$. Without loss of generality, in this proof we assume that in Partition the value of $E$ is at least 2 . 
Define $n:=r+2, \tau:=E^{2}+1, p_{j}:=2 e_{j} E, j=1,2, \ldots, r, p_{r+1}:=2 E^{2}+4$ and $p_{r+2}:=3$. We show that for the constructed instance a preemptive schedule $S$ with four moves such that $K_{\max }(S) \leq 4 E^{2}+5.5$ exists if and only if PARTition has a solution. Notice that $p(N)=6 E^{2}+7>4 \tau$, as required.

Suppose that PARTition has a solution, and $R_{1}$ and $R_{2}$ are the required subsets with $e\left(R_{1}\right)=e\left(R_{2}\right)=E$. Define a schedule $S_{4}(k)$ as in Figure 8 with $Z_{1}=R_{1} \cup\{r+1\}, Z_{2}=R_{2}$ and $k=r+2$ split by $x_{k}=y_{k}=1.5$. It is easy to verify that $K_{\max }\left(S_{4}(k)\right)=y_{r+2}+4 \tau=$ $4 E^{2}+5.5$.

Now let a schedule $S$ with four moves and with $K_{\max }(S) \leq 4 E^{2}+5.5$ exist with $Z_{1}$ and $Z_{2}$ being the sets of the whole jobs assigned to machine $M_{1}$ and $M_{2}$, respectively. In this schedule job $r+1$ cannot be fractional, since otherwise due to (5), $K_{\max }(S)>p_{r+1}+2 \tau=4 E^{2}+6$. Thus, job $r+1$ belongs to $Z_{1}$. Due to (13), the total length $Y$ of all fractional jobs on machine $M_{2}$ is at most 1.5 time units.

For schedule $S$, suppose that $p\left(Z_{2}\right)>2 \tau$. Since $K_{\max }(S) \geq \tau+Y+p\left(Z_{2}\right)+\tau$, we deduce that $Y+p\left(Z_{2}\right) \leq 2 E^{2}+3.5$. If $Y \geq 1$, then since $p\left(Z_{2}\right)$ is integer, we have that actually $p\left(Z_{2}\right) \leq 2 E^{2}+2=2 \tau$, a contradiction. On the other hand, if $0<Y<1$, then due to integrality of $p\left(Z_{2}\right)$, we derive that $p\left(Z_{2}\right)=2 E^{2}+3$. If job $r+2$ does not belong to $Z_{2}$, then there exists a non-negative integer $x$ such that $p\left(Z_{2}\right)=2 E(E+x)$. However, the equality $2 E(E+x)=2 E^{2}+3$ implies that $x=3 /(2 E)<1$, which is impossible. Otherwise, if job $r+2$ belongs to $Z_{2}$, we deduce that $Z_{2}=\{r+2\} \cup R_{2}$, where $p\left(R_{2}\right)=2 E^{2}$, i.e., the sets $R_{2}$ and $R_{1}=\{1,2, \ldots, r\} \backslash R_{2}$ form a solution to PARTition.

From now on, assume that $p\left(Z_{2}\right) \leq 2 E^{2}+2=2 \tau$. If $p\left(Z_{2}\right)<2 E^{2}$ then the total load on machine $M_{1}$ becomes $p(N)-Y-p\left(Z_{2}\right)>6 E^{2}+7-1.5-2 E^{2}=4 E^{2}+5.5$. Thus, $2 E^{2} \leq p\left(Z_{2}\right) \leq 2 E^{2}+2$.

If set $Z_{2}$ contains job $r+2$, then, since the processing time of any job except $r+2$ is an even number, if follows that $p\left(Z_{2}\right)$ is odd, so that in fact $p\left(Z_{2}\right)=2 E^{2}+1$. This means that for some positive integer $x$ we have $p\left(Z_{2}\right)=2 E(E-x)+3=2 E^{2}+1$, which yields $x=\frac{1}{E}<1$, a contradiction.

Now, if set $Z_{2}$ does not contain job $r+2$, then for some non-negative integer $x$ we have $p\left(Z_{2}\right)=2 E(E+x) \leq 2 E^{2}+2$, which yields $x \leq \frac{1}{E}$, and since $x$ is integer we deduce that $x=0$. As above, we can define $R_{2}:=Z_{2}$ and $R_{1}:=\{1,2, \ldots, r\} \backslash R_{2}$ and obtain a solution to Partition. This proves the theorem.

The following algorithm uses the FPTAS for the subset-sum problem for finding a solution to problem $T P 2|v=1, c \geq n| K_{\max }$ that is as close to the optimum as desired.

\section{Algorithm MoveEven}

Step 1. Compute $T$ in accordance with (1).

Step 2. Given an $\varepsilon>0$, run an FPTAS for the subset-sum problem of the following structure

$$
\begin{array}{ll}
\max & \sum_{j \in N} p_{j} x_{j} \\
& \sum_{j \in N} p_{j} x_{j} \leq T \\
& x_{j} \in\{0,1\}, j \in N .
\end{array}
$$

For a found solution of problem (17), determine $Z_{1}^{\prime}:=\left\{j \in N \mid x_{j}^{\varepsilon}=1\right\}$ and $Z_{2}^{\prime}:=$ $\left\{j \in N \mid x_{j}^{\varepsilon}=0\right\}$ and create schedule $S_{2}^{\prime}$ shown in Figure 3 with $Z_{1}=Z_{1}^{\prime}$ and $Z_{2}=Z_{2}^{\prime}$. If $p\left(Z_{1}^{\prime}\right) \geq(1-\varepsilon) T$, then go to Step 7 . 


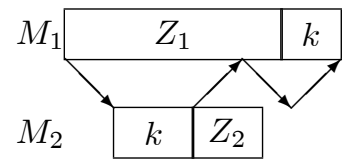

Figure 8: Schedule $S_{4}^{\prime \prime}$ with four moves and $y_{k}=y^{*}$

Step 3. Given an $\varepsilon>0$, run an FPTAS for the subset-sum problem of the following structure

$$
\begin{array}{cl}
\max & \sum_{j \in N} p_{j} x_{j} \\
& \sum_{j \in N} p_{j} x_{j} \leq T-2 \tau \\
& x_{j} \in\{0,1\}, j \in N,
\end{array}
$$

For a found solution of problem (18), determine $Z_{2}^{\prime \prime}:=\left\{j \in N \mid x_{j}^{\varepsilon}=1\right\}$ and $Z_{1}^{\prime \prime}:=$ $\left\{j \in N \mid x_{j}^{\varepsilon}=0\right\}$. Create schedule $S_{2}^{\prime \prime}$ shown in Figure 3 with $Z_{1}=Z_{1}^{\prime \prime}$ and $Z_{2}=Z_{2}^{\prime \prime}$. If $p\left(Z_{2}^{\prime \prime}\right) \geq(1-\varepsilon)(T-2 \tau)$, then go to Step 7 .

Step 4. Find set $B_{2}:=\left\{j \in N \mid p_{j}+2 \tau>T\right\}$ of the jobs of rank 2. If either $p\left(Z_{1}^{\prime \prime}\right) \leq 4 \tau$ or $p\left(B_{2}\right) \geq T$, then go to Step 7; otherwise go to Step 5 .

Step 5. If $p\left(Z_{2}^{\prime \prime}\right) \geq 2 \tau$, select an arbitrary job $k \in Z_{1}^{\prime \prime} \backslash B_{2}$, define $y_{k}:=(T-2 \tau)-p\left(Z_{2}^{\prime \prime}\right)$ and $x_{k}:=p_{k}-y_{k}$, make a schedule $S_{4}^{\prime}$ with four moves as shown in Figure 1(c) with $Z_{1}=Z_{1}^{\prime \prime} \backslash\{k\}, Z_{2}=Z_{2}^{\prime \prime}$ and go to Step 7; otherwise go to Step 6 .

Step 6 Compute $y^{*}$ by formula (15) with $W_{2}^{*}=Z_{2}^{\prime \prime}$. Select an arbitrary job $k \in Z_{1}^{\prime \prime} \backslash B_{2}$, define $y_{k}:=y^{*}$, and $x_{k}:=p_{k}-y_{k}$, make a schedule $S_{4}^{\prime \prime}$ with four moves as shown in Figure 8 with $Z_{1}=Z_{1}^{\prime \prime} \backslash\{k\}, Z_{2}=Z_{2}^{\prime \prime}$ and go to Step 7 .

Step 7. Output the best of the found schedules as schedule $S^{\varepsilon}$.

It is clear that Algorithm MoveEven requires $O(n / \varepsilon)$ time. Below we analyze its performance.

Theorem 6 For problem TP2 $|v=1, c \geq n| K_{\max }$, Algorithm MoveEven behaves as an FP$T A S$, provided that there exists an optimal schedule $S^{*}$ either with two or with four moves.

Proof: Suppose first that in an optimal schedule $S^{*}$ the transporter makes two moves.

Consider schedule $S_{2}^{\prime}$ found in Step 2. Notice that $p\left(Z_{1}^{\prime}\right) \leq T$. We have that $K_{\max }\left(S_{2}^{\prime}\right)=$ $\max \left\{p\left(Z_{1}^{\prime}\right), p\left(Z_{2}^{\prime}\right)+2 \tau\right\}$. If $p\left(Z_{1}^{\prime}\right) \geq(1-\varepsilon) T$, then we derive $p\left(Z_{2}^{\prime}\right)+2 \tau \leq 2 T-(1-\varepsilon) T=$ $(1+\varepsilon) T$. Thus, due to $(2)$ we obtain that $K_{\max }\left(S_{2}^{\prime}\right) \leq(1+\varepsilon) T \leq(1+\varepsilon) K_{\max }\left(S^{*}\right)$.

Similarly, for schedule $S_{2}^{\prime \prime}$ found in Step 3, notice that $p\left(Z_{2}^{\prime \prime}\right)+2 \tau \leq T$. We have that $K_{\max }\left(S_{2}^{\prime \prime}\right)=\max \left\{p\left(Z_{1}^{\prime \prime}\right), p\left(Z_{2}^{\prime \prime}\right)+2 \tau\right\}$. If $p\left(Z_{2}^{\prime \prime}\right) \geq(1-\varepsilon)(T-2 \tau)$, then we derive $p\left(Z_{1}^{\prime \prime}\right)=$ $p(N)-p\left(Z_{2}^{\prime \prime}\right)=2(T-\tau)-p\left(Z_{2}^{\prime \prime}\right) \leq 2(T-\tau)-(1-\varepsilon)(T-2 \tau)=(1+\varepsilon) T-2 \varepsilon \tau$. Thus, due to $(2)$ we obtain that $K_{\max }\left(S_{2}^{\prime \prime}\right) \leq(1+\varepsilon) T \leq(1+\varepsilon) K_{\max }\left(S^{*}\right)$.

If for schedule $S_{2}^{\prime}$ found in Step 2 the inequality $p\left(Z_{1}^{\prime}\right)<(1-\varepsilon) T$ holds, then due to Theorem 3, the solution found by the FPTAS is an optimal solution to problem (17). This 
means that the value $p\left(Z_{1}^{\prime}\right)$ cannot be enlarged and the value $p\left(Z_{2}^{\prime}\right)+2 \tau$ cannot be reduced, as long as we require $p\left(Z_{1}^{\prime}\right) \leq T$, i.e., $K_{\max }\left(S^{*}\right) \geq p\left(Z_{2}^{\prime}\right)+2 \tau$, provided that there are two moves in schedule $S^{*}$.

Similarly, if for schedule $S_{2}^{\prime \prime}$ found in Step 3 the inequality $p\left(Z_{2}^{\prime \prime}\right)<(1-\varepsilon)(T-2 \tau)$ holds, then due to Theorem 3 , the solution found by the FPTAS is an optimal solution to problem (18). This means that the value $p\left(Z_{2}^{\prime \prime}\right)+2 \tau$ cannot be enlarged and the value $p\left(Z_{1}^{\prime \prime}\right)$ cannot be reduced, as long as we require $p\left(Z_{2}^{\prime \prime}\right) \leq T-2 \tau$, i.e., $K_{\max }\left(S^{*}\right) \geq p\left(Z_{1}^{\prime \prime}\right)$. The algorithm outputs schedule $S^{\varepsilon}$ such that $K_{\max }\left(S^{\varepsilon}\right) \leq \min \left\{K_{\max }\left(S_{2}^{\prime}\right), K_{\max }\left(S_{2}^{\prime \prime}\right)\right\}=\min \left\{p\left(Z_{2}^{\prime}\right)+2 \tau, p\left(Z_{1}^{\prime \prime}\right)\right\}$, which is optimal, provided that there are two moves in schedule $S^{*}$.

Thus, if there are two moves in an optimal schedule, Algorithm MoveEven outputs a schedule of a length that is at most $1+\varepsilon$ times the optimum.

The conditions of Step 4 describe situations in which the algorithm still outputs the best of the two-move schedules found so far, and such a schedule is optimal. If $p\left(Z_{1}^{\prime \prime}\right) \leq 4 \tau$ then no schedule with four moves can be shorter than $K_{\max }\left(S_{2}^{\prime \prime}\right)=p\left(Z_{1}^{\prime \prime}\right)$, i.e., for an optimal schedule with an even number of moves the equality $K_{\max }\left(S^{*}\right)=\min \left\{K_{\max }\left(S_{2}^{\prime}\right), K_{\max }\left(S_{2}^{\prime \prime}\right)\right\}$ holds. For the set $B_{2}$ of rank 2 jobs found in Step 4 suppose that $p\left(B_{2}\right) \geq T$. If in schedule $S^{*}$ each job of set $B_{2}$ is processed only on machine $M_{1}$, then $K_{\max }\left(S^{*}\right) \geq p\left(B_{2}\right)$. In this case, schedule $S_{2}^{\prime \prime}$ found in Step 3 is optimal, since no job of rank 2 may belong to set $Z_{2}^{\prime \prime}$, so that $Z_{2}^{\prime \prime}=N \backslash B_{2}, Z_{1}^{\prime \prime}=B_{2}$ and $K_{\max }\left(S_{2}^{\prime \prime}\right)=p\left(B_{2}\right)$. If in $S^{*}$ a job of rank 2 is processed, even partly, on machine $M_{2}$ then due to (5) we deduce that $K_{\max }\left(S^{*}\right) \geq p_{u}+2 \tau$, where $u \in B_{2}$ is the shortest rank 2 job. In this case, schedule $S_{2}^{\prime}$ found in Step 2 is optimal, since $Z_{1}^{\prime}=N \backslash\{u\}, Z_{2}^{\prime}=\{u\}$ and $K_{\max }\left(S_{2}^{\prime}\right)=p_{u}+2 \tau$.

In the rest of the proof, it is assumed that there are four moves in an optimal schedule $S^{*}$. We arrive at Step 5 if $p\left(Z_{1}^{\prime \prime}\right)>4 \tau$ and $p\left(B_{2}\right)<T$. Consider the sets $Z_{1}^{\prime \prime}$ and $Z_{2}^{\prime \prime}$ found in Step 3. Since no rank 2 job belongs to set $Z_{2}^{\prime \prime}$, we deduce that $B_{2} \subset Z_{1}^{\prime \prime}$ and there exists a job $k \in Z_{1}^{\prime \prime} \backslash B_{2}$ such that $p_{k}+2 \tau<T$.

If $p\left(Z_{2}^{\prime \prime}\right)>2 \tau$, then due to Lemma 4 , we can create schedule $S_{4}^{\prime}$ shown in Figure 1(c) with $K_{\max }\left(S_{4}^{\prime}\right)=T$, which means this schedule is optimal; see Step 5.

On the other hand, if $p\left(Z_{2}^{\prime \prime}\right) \leq 2 \tau$, then $p\left(Z_{1}^{\prime \prime}\right)=p(N)-p\left(Z_{2}^{\prime \prime}\right) \geq(2 T-2 \tau)-2 \tau=2 T-4 \tau$. Recall that the FPTAS in Step 3 solves the corresponding subset-sum problem optimally, so that for the value $y^{*}$ found by formula (15) with $W_{2}^{*}=Z_{2}^{\prime \prime}$ the lower bound $K_{\max }\left(S^{*}\right) \geq y^{*}+4 \tau$ holds. Notice that

$$
y^{*}=\frac{p\left(Z_{1}^{\prime \prime}\right)}{2}-2 \tau \text {. }
$$

so that it follows from $p\left(Z_{1}^{\prime \prime}\right)>4 \tau$ that $y^{*}>0$. Since the solution found by the FPTAS is an optimal solution to problem (18), it follows that for any job $k \in Z_{1}^{\prime \prime}$ the inequality $p\left(Z_{2}^{\prime \prime}\right)+p_{k}>T-2 \tau$ holds, which is equivalent to $p\left(Z_{1}^{\prime \prime}\right)-p_{k}<T$. Thus, we derive from $p\left(Z_{1}^{\prime \prime}\right) \geq 2 T-4 \tau$ that

$$
p_{k}>p\left(Z_{1}^{\prime \prime}\right)-T=y^{*}+\frac{p\left(Z_{1}^{\prime \prime}\right)}{2}+2 \tau-T \geq y^{*} .
$$

In Step 6, the algorithm selects a job $k \in Z_{1}^{\prime \prime} \backslash B_{2}$ and assigns it to be processed on machine $M_{2}$ for $y^{*}$ time units, where as proved above, $0<y^{*}<p_{k}$. For job $k \in Z_{1}^{\prime \prime} \backslash B_{2}$, we derive

$$
p\left(Z_{1}^{\prime \prime}\right)-p_{k}=\frac{p\left(Z_{1}^{\prime \prime}\right)}{2}+\frac{p\left(Z_{1}^{\prime \prime}\right)}{2}-p_{k} \geq \frac{p\left(Z_{1}^{\prime \prime}\right)}{2}+(T-2 \tau)-(T-2 \tau)=\frac{p\left(Z_{1}^{\prime \prime}\right)}{2} .
$$

In schedule $S_{4}^{\prime \prime}$ job $k$ is delivered back to machine $M_{1}$ at time $2 \tau+y^{*}=\frac{p\left(Z_{1}^{\prime \prime}\right)}{2}<p\left(Z_{1}^{\prime \prime}\right)-p_{k}$, so that the structure of schedule $S_{4}^{\prime \prime}$ is as shown in Figure 8 with $Z_{1}=Z_{1}^{\prime \prime} \backslash\{k\}$. Since 
$K_{\max }\left(S_{4}^{\prime \prime}\right)=y^{*}+4 \tau=p\left(Z_{1}^{\prime \prime}\right)-y^{*}=\frac{p\left(Z_{1}^{\prime \prime}\right)}{2}+2 \tau$, this schedule is optimal due to (14).

Notice that if an instance of the problem contains a job of rank 0, Algorithm MoveEven outputs schedule $S_{2}^{\prime \prime}$ as an optimal schedule. Indeed, for a rank 0 job $k$ we have that $p_{k} \geq T$, so that $p(N \backslash\{k\}) \leq T-2 \tau$ and in Step 3 we will have $Z_{1}^{\prime \prime}=\{k\}, Z_{2}^{\prime \prime}=p(N \backslash\{k\})$. Such a schedule is shown in Figure $3(\mathrm{a})$. This observation allows us to exclude from further consideration the instances of the problem with a rank 0 job, since for the purpose of finding a global optimal solution there is no need to create schedules with more than two moves.

\section{Finding the Best Schedule with Three Moves}

In this section, we show that for problem $T P 2|v=1, c \geq n| K_{\max }$ finding the best schedule with three moves is an NP-hard problem and give an algorithm that for many instances finds an exact solution, but in general behaves as an FPTAS.

In this section we refer to a rank 3 job as long; otherwise, if a job does not satisfy (6) for $r=3$, it is called short. As seen from the consideration below, the presence of the short jobs is crucial for fast finding an exact solution to the problem.

As proved in Lemma 8, the search for the best schedule with three moves can be limited to (i) non-preemptive schedules with the closest possible loads on the machines (see Figure 4), or (ii) a preemptive schedules with a single fractional job (see Figure 2), for which the value of the objective function meets a lower bound.

First, we prove that under some conditions finding such a schedule is an NP-hard problem.

Theorem 7 For problem $T P 2|v=1, c \geq n| K_{\max }$ with no short jobs and $p(N) \geq 4 \tau$, finding the best non-preemptive schedule with three moves is NP-hard in the ordinary sense.

Proof: As in the previous NP-hardness proofs, we reduce PARTITION to the decision version of problem $T P 2|v=1, c \geq n| K_{\max }$.

Define $n=r, \tau=E$ and $p_{j}=2 e_{j}, j=1,2, \ldots, n$. We show that for the constructed instance a non-preemptive schedule $S$ with three moves and such that $K_{\max }(S) \leq 3 E$ exists if and only if PARTition has a solution. Notice that $p(N)=4 E=4 \tau$, as required.

Suppose that Partition has a solution, and $R_{1}$ and $R_{2}$ are the required subsets with $e\left(R_{1}\right)=e\left(R_{2}\right)=E$. Define a schedule $S$ as in Figure 4 (a) with $Z_{1}=R_{1}$. It is easy to verify that $K_{\max }\left(S_{3}\right)=3 E$.

Suppose that a non-preemptive schedule $S$ with three moves and with $K_{\max }(S) \leq 3 E$ exists. The first (downward) move starts at time zero, the second (upward) move starts at time $\tau=E$, and the final (downward) move starts at $2 E$ and completes at $3 E$. All jobs assigned to machine $M_{1}$ are processed in the time interval $[0,2 E]$, while those assigned to machine $M_{2}$ are processed in the interval $[E, 3 E]$. If $R_{i}$ denotes the set of the jobs of set $\{1,2, \ldots, n\}$ assigned to machine $M_{i}$, for $i \in\{1,2\}$, then the sets $R_{1}$ and $R_{2}$ form a solution to Partition. This proves the theorem.

Theorem 8 For problem $T P 2|v=1, c \geq n| K_{\max }$ with no short jobs and $p(N) \geq 4 \tau$, finding the best preemptive schedule with three moves is NP-hard in the ordinary sense.

Proof: As in the previous NP-hardness proofs, we reduce PARTITION to the decision version 
of problem $T P 2|v=1, c \geq n| K_{\max }$. Without loss of generality, in this proof we assume that in Partition the value of $E$ is at least 3 .

Define $n=r+3, \tau=E^{2}+1, p_{j}=2 e_{j} E, j=1,2, \ldots, r, p_{r+1}=p_{r+2}=p_{r+3}=3$. We show that for the constructed instance a preemptive schedule $S$ with three moves such that $K_{\max }(S) \leq 3 E^{2}+6$ exists if and only if PARTition has a solution.

Notice that $p(N)=4 E^{2}+9>4 \tau$, as required. Besides, there is no short job, since $p_{j} \geq 3$ for all $j \in N$, so that $p_{j}+3 \tau \geq 3+3 \tau=3 E^{2}+6>T=\left(4 E^{2}+9\right) / 2+E^{2}+1=3 E^{2}+5.5$.

Suppose that PARtition has a solution, and $R_{1}$ and $R_{2}$ are the required subsets with $e\left(R_{1}\right)=e\left(R_{2}\right)=E$. Define a schedule $S_{3}(k)$ as in Figure $2(\mathrm{~b})$ with $Z_{1}=R_{1} \cup\{r+1\}$, $Z_{2}=R_{2} \cup\{r+2\}$ and $k=r+3$ being the fractional job with $x_{r+3}=y_{r+3}=1.5$. Since $y_{r+3}+2 \tau>p\left(Z_{1}\right)$ and $x_{r+3}+2 \tau>p\left(Z_{2}\right)$, we deduce that $K_{\max }\left(S_{3}(k)\right)=p_{r+3}+3 \tau=3 E^{2}+6$.

Now let a preemptive schedule $S$ with three moves and with $K_{\max }(S) \leq 3 E^{2}+6$ exist. Due to Lemma 8 exactly one job $k$ is fractional and $K_{\max }(S) \geq p_{k}+3 \tau$. This implies that the fractional job is one of the jobs of the set $\{r+1, r+2, r+3\}$. Without loss of generality, assume that job $k=r+3$ is fractional, and it is processed on machine $M_{1}$ during $x_{k}$ time units and on machine $M_{2}$ during $y_{k}=3-x_{k}$ time units. Additionally assume that $p\left(Z_{2}\right) \leq p\left(Z_{1}\right)$. If either $p\left(Z_{1}\right)>2 \tau+y_{k}$ or $p\left(Z_{2}\right)>2 \tau+x_{k}$, then we would have $K_{\max }(S) \geq \max \left\{p\left(Z_{1}\right)+x_{k}, p\left(Z_{2}\right)+y_{k}\right\}+\tau>3 \tau+p_{r+3}=3 E^{2}+6$, which is impossible. Thus, $p\left(Z_{2}\right) \leq \frac{1}{2}\left(p\left(Z_{1}\right)+p\left(Z_{2}\right)\right) \leq \frac{1}{2}\left(4 \tau+y_{k}+x_{k}\right)=2 \tau+1.5=2 E^{2}+3.5$. Since for each job of set $Z_{2}$ the processing time is integer, we obtain that actually $p\left(Z_{2}\right) \leq 2 E^{2}+3$. Set $Z_{2}$ contains $l$ jobs of length 3 , where $l \in\{0,1,2\}$ (i.e., some or none of jobs $r+1$ and $r+2)$. For $l \in\{0,1\}$ we have that for some non-negative integer $x$ the inequality $p\left(Z_{2}\right)=2 E(E+x)+3 l \leq 2 E^{2}+3$ holds, which yields $x \leq \frac{3-3 l}{2 E}<1$. For $l=2$ we have that $p\left(Z_{2}\right)=2 E(E-x)+6$ for some non-negative integer $x$, and $p\left(Z_{1}\right)=p(N)-p_{k}-p\left(Z_{2}\right)=$ $2 E(E+x)$. Since $K_{\max }(S) \geq p\left(Z_{1}\right)+\tau$, we deduce $2 E(E+x) \leq 2 E^{2}+5$, so that $x \leq \frac{5}{2 E}<1$. Since $x$ must be integer, we conclude that $x=0$. If the set $R_{i}$ denotes the set of the jobs of set $\{1,2, \ldots, r\}$ assigned to machine $M_{i}$, for $i \in\{1,2\}$, then the sets $R_{1}$ and $R_{2}$ form a solution to Partition. This proves the theorem.

Algorithm Move3 presented below finds the best schedule with three moves for problem $T P 2|v=1, c \geq n| K_{\max }$.

\section{Algorithm Move3Try}

Step 1. Compute $T$ in accordance with (1).

Step 2. Take an $\varepsilon>0$ and run an FPTAS for the subset-sum problem of the following structure

$$
\begin{aligned}
\max & \sum_{j \in H} p_{j} x_{j} \\
& \sum_{j \in H} p_{j} x_{j} \leq T-\tau \\
& x_{j} \in\{0,1\}, j \in H,
\end{aligned}
$$

where $H=N$. For a found solution of problem (??), define $H^{(1)}=\left\{j \in H \mid x_{j}^{\varepsilon}=1\right\}$.

Step 3. Create a non-preemptive schedule $S_{3}$ shown in Figure 4 with $Z_{1}=H^{(1)}$. If $p\left(H^{(1)}\right)<$ $(1-\varepsilon)(T-\tau)$, then go to Step 4 .

Step 4. Determine set $B:=\left\{j \in N \mid p_{j} \geq 2 \varepsilon(T-\tau)\right\}$. Let $|B|=h$. Renumber the jobs of set $B$ so that $p_{1} \leq p_{2} \leq \ldots \leq p_{h}$. Define $\underline{k}:=1$ and $\bar{k}:=h$.

(a) Compute $k=\lceil(\bar{k}+\underline{k}) / 2\rceil$. If $\underline{k}=\bar{k}$, then go to Step 5 . 
(b) Define $N_{k}=N \backslash\{k\}$. Compute $\varepsilon_{k}=p_{k} /(T-\tau)$, take $\varepsilon=\varepsilon_{k}$ and apply an FPTAS to the subset-sum problem of the form (??) with $H=N_{k}$. For a found solution of problem (??), define $H^{(1)}=\left\{j \in H \mid x_{j}^{\varepsilon}=1\right\}$.

(c) If $p\left(H^{(1)}\right) \geq\left(1-\varepsilon_{k}\right)(T-\tau)$, then define $\bar{k}:=k$; otherwise define $\underline{k}:=k$. Return to Step $7(\mathrm{a})$.

Step 5. For the current value of $k$, define $Z_{1}:=H^{(1)}$ and $Z_{2}:=N \backslash\left(Z_{1} \cup\{k\}\right)$. Create a schedule $S_{3}(k)$ shown in Figure 2(b).

The statements below analyze the performance of Algorithm Move3.

Theorem 9 For, problem $T P 2|v=1, c \geq n| K_{\max }$ for finding the best candidate schedule with three moves Algorithm Move3 behaves as an FPTAS that requires at most $O((n \log n) / \varepsilon)$ time.

Proof: If in Step 3, $p\left(H^{(1)}\right) \geq(1-\varepsilon)(T-\tau)$, then $p\left(H^{(1)}\right) \leq T-\tau \leq p\left(N \backslash Z_{1}\right) \leq$ $(1+\varepsilon)(T-\tau)$ and $K_{\max }\left(S_{3}\right)=\max \left\{\tau+p\left(N \backslash Z_{1}\right), 3 \tau\right\}$, i.e., schedule $S_{3}$ is either optimal with $K_{\max }\left(S_{3}\right)=3 \tau$ or $K_{\max }\left(S_{3}\right)=\tau+p\left(N \backslash Z_{1}\right) \leq(1+\varepsilon) T-\varepsilon \tau \leq(1+\varepsilon) K_{\max }\left(S^{*}\right)$.

If

$$
p\left(H^{(1)}\right)<(1-\varepsilon)(T-\tau)
$$

then due to Theorem 3, the value $p\left(H^{(1)}\right)$ is optimal for the corresponding subset-sum problem of the form (??), i.e., $p\left(H^{(1)}\right) \geq p(X)$ for any set $X \subset H$ such that $p(X) \leq T-\tau$. Thus, in this case schedule $S_{3}$ is the best non-preemptive schedule with three moves.

A possibly better schedule can be found among the preemptive schedules. Recall that in such a preemptive schedule $S_{3}(k)$ there is exactly one fractional job $k$; see Figure 2 . Below we show that under the condition (20), the duration $p_{k}$ of the fractional job cannot be smaller than $2 \varepsilon(T-\tau)$.

Indeed, suppose that for schedule $S_{3}(k)$, the sets of the whole jobs on machine $M_{i}$ is denoted by $Z_{i}, i \in\{1,2\}$. The fractional job is split between the machines so that $p_{k}=x_{k}+y_{k}$, where

$$
=p\left(Z_{2}\right)+y_{k}=T-\tau \text {. }
$$

The latter equation implies that $\max \left\{p\left(Z_{1}\right), p\left(Z_{2}\right)\right\}<T-\tau$. Since $p\left(H^{(1)}\right) \geq$ $\max \left\{p\left(Z_{1}\right), p\left(Z_{2}\right)\right\}$, it follows from (20) that

$$
\max \left\{p\left(Z_{1}\right), p\left(Z_{2}\right)\right\}<(1-\varepsilon)(T-\tau),
$$

which leads to $p_{k}>2 \varepsilon(T-\tau)$.

In a preemptive schedule $S_{3}(k)$ with the fractional job $k$ either $K_{\max }\left(S_{3}(k)\right)=T$ or, if job $k$ is a Rank 3 job, the length $K_{\max }\left(S_{3}(k)\right)$ is given by $p_{k}+3 \tau$. Thus, we need to find the shortest $k$ with $p_{k} \geq 2 \varepsilon(T-\tau)$ that can be fractional in schedule $S_{3}(k)$. This is done by the binary search procedure that is outlined in Step 4 . Notice only that job $k$ can be fractional, for which $p\left(H^{(1)}\right)+p_{k} \geq(T-\tau)$ for the corresponding set $H^{(1)}$.

To justify the binary search to be performed in Step 4, we apply the following reasoning. If in some iteration of the search procedure for the current job $k$ we find the set $H^{(1)}=H_{k}^{(1)}$ such that $p\left(H_{k}^{(1)}\right)<\left(1-\varepsilon_{k}\right)(T-\tau)$, then due to the choice of $\varepsilon_{k}$ the inequality $p\left(H_{k}^{(1)}\right)+p_{k}<$ 
$T-\tau$ holds, so that job $k$ cannot be fractional. In this case, we continue our search among those jobs that are longer than $p_{k}$. To show that the required fractional job cannot be found among the jobs with indices less than $k$, assume that for some $k^{\prime}<k$ the set $H^{(1)}=H_{k^{\prime}}^{(1)}$ is found such that $p\left(H_{k^{\prime}}^{(1)}\right) \geq\left(1-\varepsilon_{k^{\prime}}\right)(T-\tau)$, which implies that $p\left(H_{k^{\prime}}^{(1)}\right)+p_{k^{\prime}} \geq T-\tau$, so that job $k^{\prime}$ can be fractional. Since $p_{k} \geq p_{k^{\prime}}$, we derive that $p\left(H_{k^{\prime}}^{(1)}\right)>p\left(H_{k}^{(1)}\right)$. The latter inequality is impossible if $k \notin H_{k^{\prime}}^{(1)}$, since $p\left(H_{k}^{(1)}\right) \geq p(X)$ for any set $X \subseteq N \backslash\{k\}$. On the other hand, if $k \in H_{k^{\prime}}^{(1)}$ then we derive that $p\left(H_{k}^{(1)}\right)<p\left(\left(H_{k^{\prime}}^{(1)} \cup\left\{k^{\prime}\right\} \backslash\{k\}\right)\right)$, again a contradiction.

If for the current job $k$, the set $H^{(1)}$ is such that $p\left(H^{(1)}\right) \geq\left(1-\varepsilon_{k}\right)(T-\tau)$, then job $k$ can be fractional and we need to consider only those jobs that are shorter than $p_{k}$. Eventually in at most $O(\log h)$ iterations the shortest possible fractional job will be found.

For the found job $k$, in Step 5 schedule $S_{3}(k)$ is found with the split $p_{k}=x_{k}+y_{k}$ of job $k$ defined by (??). If job $k$ is a Rank 3 job, i.e., $p_{k} \geq T-3 \tau$, we deduce from $p\left(Z_{1}\right)+x_{k}=T-\tau$ that $p\left(Z_{1}\right)+p_{k}=T-\tau+y_{k}$, and therefore $2 \tau+y_{k}>p\left(Z_{1}\right)$; similarly, $2 \tau+x_{k}>p\left(Z_{2}\right)$. Thus, in this case, schedule $S_{3}(k)$ is as shown in Figure 2(b), and $K_{\max }\left(S_{3}(k)\right)=p_{k}+3 \tau$. This schedule is optimal due to the choice of job $k$. If job $k$ has a smaller rank, i.e., $p_{k}<T-3 \tau$, then both inequalities $2 \tau+y_{k} \leq p\left(Z_{1}\right)$ and $2 \tau+x_{k} \leq p\left(Z_{2}\right)$ hold, i.e., schedule $S_{3}(k)$ is as shown in Figure $2(\mathrm{a})$, and $K_{\max }\left(S_{3}(k)\right)=T$.

The best of all found schedules is the best candidate schedule with three moves. Step 4 involves sorting which requires $O(h \log h)$ time and $O(\log h)$ applications of an FPTAS. Since each time the FPTAS is run with $\varepsilon_{k} \geq 2 \varepsilon$, the running time for each run does not exceed $O(n / \varepsilon)$. Thus, the overall running time of running Algorithm Move3 does not exceed $O((n \log n) / \varepsilon)$.

Thus, Algorithm Move3 behaves as an FPTAS, provided that there exists an optimal schedule with three moves, and finds an optimal schedule in some cases.

The general algorithm for handling problem $T P 2|v=1, c \geq n| K_{\max }$ involves the following stages:

1. Create a schedule $S_{0}$ in which all jobs are processed on machine $M_{1}$.

2. Run Algorithm MoveEven.

3. Run Algorithm Move3.

4. Output the best of all found schedules.

It follows from the results of Sections 3 and 4 that for some instances of the problem the final algorithm will find an optimal schedule, while for others it will behave as an FPTAS. The running time of the algorithm does not exceed $O(n \log n+n / \varepsilon)$.

\section{Non-Preemptive Schedules}

In this section, for problem $T P 2|v=1, c \geq n| K_{\max }$ we study the quality of the non-preemptive schedule compared with that of the global optimal schedule. We exhibit an instance of the problem for which the length of a non-preemptive schedule cannot be less than $\frac{4}{3}$ times the optimal length and present an algorithm that delivers the best possible non-preemptive 
schedule. In Step 3 the algorithm outputs a schedule with two moves and in Step 4 it outputs a schedule with three moves. The length of the best of all found schedules is at most $\frac{4}{3}$ times the optimum length.

\section{Algorithm NoPmtn}

Step 1. Create a schedule $S_{0}$ in which all jobs are processed on machine $M_{1}$.

Step 2. Compute $T$ in accordance with (1).

Step 3. If there exists a job $k \in N$ such that

$$
p_{k} \geq T
$$

perform Step 3(a), otherwise perform Step 3(b).

(a) Create a schedule $S_{2}$ shown in Figure 3(a) with $Z_{1}=\{k\}$ and $Z_{2}=N \backslash\{k\}$.

(b) Take $\varepsilon=\frac{1}{3}$ and apply an FPTAS to the subset-sum problem (17). For a found solution, define $N_{1}=\left\{j \in N \mid x_{j}^{\varepsilon}=1\right\}$ and $N_{2}=N \backslash N_{1}$.

(b.1) Create a schedule $S_{2}^{\prime}$ shown in Figure 3(b) with $Z_{1}=N_{1}$ and $Z_{2}=N_{2}$.

(b.2) If $\left|N_{2}\right| \geq 2$, take an arbitrary job $q \in N_{2}$ and create a schedule $S_{2}^{\prime \prime}$ shown in Figure 3(a) with $Z_{1}=N_{1} \cup\{q\}$ and $Z_{2}=N \backslash Z_{1}$.

Step 4. If there exists a job $k \in N$ such that

$$
p_{k}+\tau \geq T
$$

perform Step 4(a), otherwise perform Step 4(b).

(a) Find a schedule $S_{3}$ with three moves shown in Figure 4 with $Z_{1}=\{k\}$ and $Z_{2}=$ $N \backslash\{k\}$.

(b) Take $\varepsilon=\frac{1}{3}$ and apply an FPTAS to the subset-sum problem (??) with $H=N$. For a found solution, define $N_{1}=\left\{j \in N \mid x_{j}^{\varepsilon}=1\right\}$ and $N_{2}=N \backslash N_{1}$. Create a schedule $S_{3}^{\prime}$ with three moves shown in Figure 4 with $Z_{1}=N_{1}$ and $Z_{2}=N_{2}$.

Step 5. Output the best of all created schedules as a heuristic schedule $S^{H}$.

The running time of Algorithm NoPmtn does not exceed $O(n)$. Below we analyze its worst-case performance.

Theorem 10 For problem $T P 2|v=1, c \geq n| K_{\max }$, Algorithm NoPmtn outputs a nonpreemptive schedule $S^{H}$ such that

$$
\frac{K_{\max }\left(S^{H}\right)}{K_{\max }\left(S^{*}\right)} \leq \frac{4}{3}
$$

For any $\varepsilon>0$ there exists an instance of problem $T P 2|v=1, c \geq n| K_{\max }$ for which the length of the best non-preemptive schedule $S$ is no less than $\left(\frac{4}{3}-\varepsilon\right) K_{\max }\left(S^{*}\right)$. 
Proof: We start with proving the bound (22) and then prove that this bound is the best possible as long as the search for a heuristic solution is limited to the class of non-preemptive schedules.

We split the proof into several parts, based on the assumption regarding the type of an optimal schedule.

Case 0. If there exists an optimal schedule in which all jobs are assigned to one machine, then schedule $S_{0}$ is optimal.

Case 1. Assume that an optimal schedule $S^{*}$ is a Type 2 schedule and analyze the schedules created in Step 3.

Suppose that there exists a rank 0 job $k \in N$. This implies that

$$
p_{k} \geq \frac{p(N)}{2}+\tau
$$

and

$$
p_{k} \geq p(N)-p_{k}+2 \tau \text {. }
$$

The latter inequality guarantees that

$$
K_{\max }\left(S_{2}\right)=\max \left\{p_{k}, p(N)-p_{k}+2 \tau\right\}=p_{k},
$$

so that $S_{2}$ is an optimal schedule due to (4).

In Step 3(b) for each job $j \in N$ the inequality

$$
p_{j}<T
$$

holds. Analyzing schedule $S_{2}^{\prime}$, we derive that

$$
K_{\max }\left(S_{2}^{\prime}\right)=\max \left\{p\left(N_{1}\right), p(N)-p\left(N_{1}\right)+2 \tau\right\} .
$$

Since $p\left(N_{1}\right) \leq T$, we only need to consider the situation that $K_{\max }\left(S_{2}^{\prime}\right)=p(N)-p\left(N_{1}\right)+$ $2 \tau$.

A possible outcome of the FPTAS is that a solution $x_{j}^{\varepsilon}, j \in N$, will be found such that

$$
\left(1-\frac{1}{3}\right) T \leq \sum_{j=1}^{n} p_{j} x_{j}^{\varepsilon}=p\left(N_{1}\right) \leq T
$$

so that

$$
\begin{aligned}
K_{\max }\left(S_{2}^{\prime}\right) & =p(N)-p\left(N_{1}\right)+2 \tau \leq p(N)-\frac{2}{3} T+2 \tau \\
& =2(T-\tau)-\frac{2}{3} T+2 \tau=\frac{4}{3} T .
\end{aligned}
$$

An alternative outcome of the application of an FPTAS with $\varepsilon=\frac{1}{3}$ to problem (17) is that the problem will be solved optimally and for the found solution $x_{j}^{\varepsilon}, j \in N$, the inequality

$$
\left(1-\frac{1}{3}\right) T>\sum_{j=1}^{n} p_{j} x_{j}^{\varepsilon}=p\left(N_{1}\right)
$$


holds. Notice that $p\left(N_{1}\right)<\frac{2}{3} T=\frac{1}{3} p(N)+\frac{2}{3} \tau$ and $p\left(N_{2}\right)>\frac{2}{3} p(N)-\frac{2}{3} \tau$. For schedule $S_{2}^{\prime \prime}$ we have that

$$
K_{\max }\left(S_{2}^{\prime \prime}\right)=\max \left\{p\left(N_{1}\right)+p_{q}, p(N)-p\left(N_{1}\right)-p_{q}+2 \tau\right\} .
$$

For any job $q \in N_{2}$, it follows that that $p_{q} \leq p\left(N_{1}\right)<\frac{2}{3} T$ and $p\left(N_{1}\right)+p_{q}>T$, so that $p_{q}+p\left(N_{1}\right) \leq \frac{4}{3} T$. If $\left|N_{2}\right|=1$, then the latter inequality implies that the bound (22) holds for $S^{H}=S_{0}$. Otherwise, if $\left|N_{2}\right| \geq 2$, then

$$
p(N)-p\left(N_{1}\right)-p_{q}+2 \tau \leq p(N)-T+2 \tau=2(T-\tau)-T+2 \tau=T,
$$

and for $S^{H}=S_{2}^{\prime \prime}$ the bound (22) holds.

Case 2. Assume that an optimal schedule $S^{*}$ is a schedule with three moves and analyze the schedules created in Step 4.

Suppose that there exists a rank 1 job $k \in N$. We deduce

$$
p_{k} \geq \frac{p(N)}{2},
$$

which is equivalent to

$$
p_{k} \geq p(N)-p_{k}
$$

The latter inequality implies that

$$
K_{\max }\left(S_{3}\right)=\max \left\{\tau+p_{k}, p(N)-p_{k}+\tau, 3 \tau\right\}=\max \left\{\tau+p_{k}, 3 \tau\right\},
$$

so that schedule $S_{3}$ is in fact optimal due to (3).

In Step 4(b) for each job $j \in N$ the inequality

$$
p_{j}+\tau<T
$$

holds, which means that

$$
p_{j}<\frac{p(N)}{2} \text {. }
$$

We show that the FPTAS will always find a solution $x_{j}^{\varepsilon}, j \in N$, such that

$$
\left(1-\frac{1}{3}\right)(T-\tau) \leq \sum_{j=1}^{n} p_{j} x_{j}^{\varepsilon} \leq T-\tau,
$$

so that

$$
\frac{2}{3}(T-\tau) \leq p\left(N_{1}\right) \leq(T-\tau) .
$$

If (24) were not true, then the instance would not contain a job $k$ such that

$$
p_{k}>\frac{2}{3}(T-\tau)
$$

otherwise, the FPTAS would assign $x_{k}^{\varepsilon}=1$ and $k \in N_{1}$.

If the outcome of the FPTAS is such that the inequality

$$
\left(1-\frac{1}{3}\right)(T-\tau)>\sum_{j=1}^{n} p_{j} x_{j}^{\varepsilon}=p\left(N_{1}\right)
$$


holds, it follows that any job $k \in N_{2}$ is such that $p\left(N_{1}\right)+p_{k}>T-\tau$. However, since $p_{k} \leq \frac{2}{3}(T-\tau)$, we derive that $p\left(N_{1}\right)+p_{k}<\frac{4}{3}(T-\tau)$. This implies that

$$
\frac{2}{3}(T-\tau) \leq p\left(N_{2} \backslash\{k\}\right)<T-\tau,
$$

and the existence of such a set $N_{2} \backslash\{k\}$ should have been identified by the FPTAS due to Theorem 3. Due to the derived contradiction, under the condition (23) the FPTAS will find a solution that satisfies (24).

Analyzing schedule $S_{3}^{\prime}$ we derive that

$$
K_{\max }\left(S_{3}^{\prime}\right)=\max \left\{p\left(N_{1}\right)+\tau, p(N)-p\left(N_{1}\right)+\tau, 3 \tau\right\}
$$

If the maximum in the right-hand side of the above equality is equal to $3 \tau$, then $S_{3}^{\prime}$ is optimal. Otherwise,

$$
p\left(N_{1}\right)+\tau \leq(T-\tau)+\tau=T
$$

and

$$
\begin{aligned}
p(N)-p\left(N_{1}\right)+\tau & \leq p(N)-\frac{2}{3}(T-\tau)+\tau \\
& =2(T-\tau)-\frac{2}{3}(T-\tau)+\tau \\
& =\frac{4}{3}(T-\tau)+\tau \leq \frac{4}{3} T .
\end{aligned}
$$

Thus, for $S^{H}=S_{3}^{\prime}$ the bound (22) holds.

We have proved that for the best of the schedules found by Algorithm NoPmtn the bound (22) holds.

To see that the bound (22) cannot be improved for a non-preemptive schedule, consider the following instance of problem $T P 2|v=1, c \geq n| K_{\max }$. There are three jobs with $p_{1}=$ $p_{2}=p_{3}=2$ and $\tau=\varepsilon$. The best non-preemptive schedule $S^{N}$ is a schedule with two moves in which two jobs are processed on machine $M_{1}$ and one job on machine $M_{2}$, so that $K_{\max }\left(S^{N}\right)=4$. The best preemptive schedule $S^{*}$ is a schedule with three moves in which one of the jobs is split between the machines, with 1 unit of processing on each machine. We have that $K_{\max }\left(S^{*}\right)=3+\varepsilon$ and

$$
\frac{K_{\max }\left(S^{N}\right)}{K_{\max }\left(S^{*}\right)}=\frac{4}{3+\varepsilon}>\frac{4}{3}-\varepsilon
$$

This completes the proof of the theorem.

\section{Conclusion}

The paper presents an algorithm for the problem on two identical parallel machines to minimize the length of a schedule, i.e., the time by which all completed jobs are collected together on board the transporter that brings all jobs to one of the machines and moves the jobs between the machines. The algorithm either solves the problem optimally, or behaves as an FPTAS for those instances that can be proved NP-hard. An important feature of the algorithm is the use of an FPTAS for the subset-sum problem that is known to delver an exact 
optimal solution under certain conditions. The paper continues the study of scheduling models with a single uncapacitated transporter, see Soper and Strusevich (2007) and Lushchakova et al. (2009).

It would be interesting to extend this approach to uniform machines, i.e., machines of different speeds. An ultimate research goal in this area will be the study of general models with more than two machines and several transporters of different capacities.

\section{ACKNOWLEDGEMENT}

The first and third authors were partly supported by the EPSRC funded project EP/I018441/1 "Quadratic and Linear Knapsack Problems with Scheduling Applications".

\section{References}

Brucker P, Knust S, Cheng TCE, Shakhlevich NV (2004) Complexity results for flow-shop and open-shop scheduling problems with transportation delays. Ann Oper Res 129: 81-106

Dell'Amico M (1996) Shop problems with two machines and time lags. Oper Res 44: 777-787

Hall LA (1998) Approximability of flow shop scheduling. Math Progr B 82: 175-190

Hurink J, Knust S (2001) Makespan minimization for flow-shop problems with transportation times and a single robot, Discrete Appl Math 112: 199-216

Kellerer H, Mansini R, Pferschy U, Speranza MG. (2003) An efficient fully polynomial approximation scheme for the Subset-Sum Problem. J Comput Syst Sci 66: 349-370.

Kellerer H, Pferschy U, Pisinger D (2004) Knapsack problems, Springer, Berlin

Kellerer H, Soper AJ, Strusevich VA (2010) Transporting jobs through a processing center with two parallel machines. In: Wu W, Daescu O (eds) COCOA 2010, Part I, Lect Notes Comput Sci 6508, pp. 408-422

Lee C-Y, Chen Z-L (2001) Machine scheduling with transportation times. J Schedul 4: 3-24

Lee C-Y, Strusevich VA (2005) Two-machine shop scheduling with an uncapacitated interstage transporter. IIE Trans 37: 725-736

Lushchakova IN, Soper AJ, Strusevich VA (2009) Transporting jobs through a two-machine open shop. Naval Res Log 56: 1-18

Qi X (2006) A logistic scheduling model: scheduling and transshipment for two processing centers. IIE Trans 38: 609-618

Soper AJ, Strusevich VA (2007) An improved approximation algorithm for the two-machine flow shop scheduling problem with an interstage transporter, Int J Found Comput Sci 18: $565-591$.

Strusevich VA (1999) A heuristic for the two-machine open-shop scheduling problem with transportation times. Discrete Appl Math 1999: 287-304.

Yu W, Hoogeveen H, Lenstra JK (2004) Minimizing makespan in a two-machine flow shop with delays and unit-time operations is NP-hard. J Schedul 7: 333-348. 